\title{
EXISTENCE AND UNIQUENESS RESULTS FOR NON-NEWTONIAN FLUIDS OF THE OLDROYD TYPE IN UNBOUNDED DOMAINS
}

\author{
RODOLFO SALVI \\ Department of Mathematics \\ Politecnico di Milano \\ P.zza L. da Vinci 32, 20133 Milan, Italy \\ E-mail: rodsal@mate.polimi.it
}

\begin{abstract}
In the paper [13], we give the full system of equations modelling the motion of a fluid/viscoelastic solid system, and obtain a differential model similar to the so-called Oldroyd model for a viscoelastic fluid. Moreover, existence results in bounded domains are obtained. In this paper we extend the results in [13] to unbounded domains. The unique solvability of the system of equations is established locally in time and globally in time with so-called smallness restrictions. Moreover, existence of a weak solution is treated.
\end{abstract}

1. Introduction. The Navier-Stokes equations

$$
\begin{aligned}
& \rho\left(\partial_{t} u+u \cdot \nabla u\right)-\nabla \cdot\left(\mu\left(\nabla u+{ }^{T} \nabla u\right)-p I\right)=\rho f \\
& \nabla \cdot u=0
\end{aligned}
$$

where $u$ is the velocity, $p$ the pressure, $\rho$ the density (constant), $\mu$ the viscosity (constant) and $\mathrm{f}$ the body force, are essentially the simplest equations describing the motion of a fluid, and they are derived under a quite simple assumption, namely, the existence of a linear local relation between tangential part of stress tensor $T$ and strain rate $D(u)=\nabla u+\nabla u^{T}$, i.e. $T=\mu D(u)$.

Physically it is assumed that the constituent particles of the fluid are too small for their dynamics to interact substantially with macroscopic motion. These fluids are called Newtonian fluids. We notice that the mathematical theory of this model is far from complete.

2000 Mathematics Subject Classification: 35Q30, 76A05, 76D03.

Key words and phrases: two-phase problem, viscoelastic material, Oldroyd model, local and global solution, weak solution.

The paper is in final form and no version of it will be published elsewhere. 
But, the Newtonian model is inadequate for fluids having a much complex chemical structure. In some materials stress increases with shear rate (shear-thickening). In most other fluids the opposite behavior takes place, the stress decreases with the increase of the stress rate (shear-thinning). This behavior is due to the progressive orientation of molecules in the motion direction. For these fluids the viscosity is considered not constant, but shear rate dependent, in particular, a nonlinear power type dependence is considered.

Anyway, by far, the most widely studied class of non-Newtonian fluids is that of viscoelastic fluids, namely, fluids with memory; see [3], [7], [8], [10]. These fluids present two typical features: stress relaxation and creep. The first phenomenon is the progressive rather than instantaneous stress decay when the fluid deformation suddenly vanishes. The second effect, dual to the former, consists in a nonlinear increasing deformation, though the fluid undergoes a constant stress. It turns out that a general constitutive law for these fluids does not exist. The choice of a model follows from rheological considerations and from accordance to experimental data. The differential models considered in literature are based on the empirical model of Maxwell that incorporate in one equation simplest shear law of viscous fluids and that of elastic solid.

In [13] we consider the motion of a continuum medium consisting of two components, namely a viscous incompressible fluid and viscoelastic particles. (In engineering science the system of fluid/elastic solid is commonly used to simulate a visco-elastic fluid). Using the theory developed in [6], [9], [12], [13], we give the full system of equations modelling the motion of the fluid/solid system, and we obtain a model like the so-called Oldroyd model. According to the scale size of the particles, we deduce two models. The discontinuous model is obtained using as order parameter the characteristic function of the region filled with the particles. The continuous model is obtained using as order parameter the volume concentration of the particles. Moreover, an existence theory is developed in bounded domains.

In this paper, we extend the results in [13] to a generic unbounded domain. Under regularity of the data, we prove some local and, for small data, global (in time) existence results. Then, assuming that initially the elastic solid is in stressed state, we prove the existence of a weak and of a weak-measure solution. Finally, an alternative model is considered.

The paper is organized as follows.

In section 2 we give notation, preliminary results and we present the systems of equations deduced in [13]. In section 3 we state the existence results. In sections 4 and 5 the existence theorems are proved.

\section{Preliminaries.}

2.1. Notation and functional spaces. Let $\Omega$ denote an open set in $R^{m}$. $\partial \Omega$ or $\Gamma$ denotes the boundary of $\Omega$. Moreover, it is assumed that $\Omega$ is a smooth domain of class $C^{k}$ with $k$ a positive integer. Furthermore, we assume that the unit normal vector field $n(x)$ with $x \in \partial \Omega$ is outward pointing on $\partial \Omega$. If it is necessary we consider also an extension of $n$ to a neighborhood of $\bar{\Omega}$. If $k \geq 2$, in each point of $x \in \partial \Omega$, we can define the mean curvature $\mathcal{H}$. 
To simplify the discussion, we do not distinguish in our notation whether the functions are $R-, R^{m}$-valued or tensors, and $c$ denotes a constant. We define $C_{0}^{\infty}(\Omega)$ to be the linear space of infinitely differentiable functions with compact supports in $\Omega$. Now let $\left(C_{0}^{\infty}(\Omega)\right)^{\prime}$ denote the dual space of $C_{0}^{\infty}(\Omega)$, the space of distributions on $\Omega$. We denote by $\langle\cdot, \cdot\rangle$ the duality pairing between $\left(C_{0}^{\infty}(\Omega)\right)^{\prime}$ and $C_{0}^{\infty}(\Omega)$.

Let $\alpha=\left(\alpha_{1}, \ldots, \alpha_{n}\right) \in N^{n}$ and set $|\alpha|=\sum_{i=1}^{n} \alpha_{i}$. We set

$$
\frac{\partial}{\partial x_{i}}=\partial_{x_{i}} \quad D^{\alpha}=\partial_{x_{1}^{\alpha_{1}, \ldots, x_{n}}}^{|\alpha|}
$$

$\nabla=\left(\partial_{x_{1}}, \ldots, \partial_{x_{m}}\right)$ the gradient operator and $\nabla \cdot$ the divergence operator.

We denote by $\mathcal{C}_{0}^{\infty}$ the linear space of divergence free functions of $C_{0}^{\infty}$. For any $s, q$, $s \geq 0, q \geq 1, \tilde{W}^{s, q}(\Omega)$ denotes the Sobolev space of order $s$ on $L^{q}(\Omega)$. Further the norm on $\tilde{W}^{s, q}(\Omega)$ is denoted by \|\|$\phi\|\|_{s, q}$.

When $q=2, \tilde{W}^{s, 2}(\Omega)$ is usually denoted by $\tilde{H}^{s}(\Omega)$ and we drop the subscript $q=2$ when referring to its norm. $\tilde{H}^{s}(\Omega)$ is a Hilbert space for the scalar product

$$
(((u, v)))_{s}=\sum_{|\alpha| \leq s} \int_{\Omega} D^{\alpha} u D^{\alpha} v d x .
$$

In particular, in $L^{2}(\Omega)$, we write the scalar product $(u, v)$ and the norm $|v|_{2}$. Furthermore, we define $\tilde{W}_{0}^{s, q}(\Omega)$ to be the closure of $C_{0}^{\infty}(\Omega)$ for the norm $\|\mid \cdot\| \|_{s, q}$.

We denote by $\tilde{W}^{-s, q^{\prime}}(\Omega)$ the dual space of $W_{o}^{s, q}(\Omega)$ and $\|\mid \cdot\| \|_{-s, q^{\prime}}$ denotes its norm where $q^{\prime}$ satisfies $1 / q+1 / q^{\prime}=1$.

Let us introduce the following spaces of divergence-free functions. We denote by

$$
\tilde{V}^{s}=\left\{v \mid v \in \tilde{H}_{0}^{s}(\Omega), \nabla \cdot v=0\right\} .
$$

Moreover, we define $H^{s}(\Omega)$ a Hilbert space for the scalar

$$
((u, v))_{s}=\sum_{|\alpha|=s} \int_{\Omega} D^{\alpha} u D^{\alpha} v d x .
$$

$V^{s}$ is the closure of $\mathcal{C}_{0}^{\infty}(\Omega)$ for the (semi-)norm $\|\cdot\|_{s}=((\cdot, \cdot))_{s}$. We set $V^{1}=V$ and $V^{0}=H$. Moreover, $P$ denotes the projection operator from $L^{2}$ onto $H$.

In general the norm in the space $\mathcal{L}$ is denoted $\|\cdot\|_{\mathcal{L}}$

Throughout the paper we need the following propositions.

Proposition 2.1 (Gagliardo-Nirenberg). Let $\Omega \subset R^{n}$ be an open set and sufficiently regular. The multiplicative inequality,

$$
\sum_{|\alpha|=r}\left|D^{\alpha} \phi\right|_{q} \leq c|\phi|_{q_{1}}^{1-\theta}\left(\sum_{|\alpha|=l}\left|D^{\alpha} \phi\right|_{q_{2}}\right)^{\theta},
$$

for $1 \leq q_{1}, q_{2} \leq \infty, 0 \leq r \leq l$,

$$
\frac{n}{q}-r=(1-\theta) \frac{n}{q_{1}}+\theta\left(\frac{n}{q_{2}}-l\right), \quad \frac{r}{l} \leq \theta \leq 1,
$$

holds with the following exceptions:

a) if $r=0, l<\frac{n}{q_{2}}$, and $q_{1}=\infty$ and $\Omega$ unbounded, we assume in addition that or $\phi \rightarrow 0$ as $x \rightarrow \infty$ or $\phi \in L^{p}$ for some $p>0$; 

$\theta=1$.

b) if $1<q_{1}<\infty$ and $l-r-\frac{n}{q_{2}}$ is a non-negative integer, then (2.1) does not hold for

The next proposition, due to Heywood [4], is based on a regularity theorem of Cattabriga [2].

Proposition 2.2. Let $\Omega$ be an open set of $R^{3}$ with boundary uniformly of class $C^{3}$. Then

$$
\begin{gathered}
\left|D^{2} v\right|_{2} \leq c\left(|A v|_{2}+|\nabla v|_{2}\right), \\
|\nabla v|_{3} \leq c\left(|A v|_{2}^{1 / 2}|\nabla v|_{2}^{1 / 2}+|\nabla v|_{2}\right), \\
\sup _{x \in \Omega}|v(x)| \leq c\left(|A v|_{2}+|\nabla v|_{2}\right),
\end{gathered}
$$

with $v \in H^{2} \cap V$ and $A=-P \Delta$ is the Stokes operator and $c$ depends only on the regularity of $\partial \Omega$ but not on the "size" of $\partial \Omega$ or $\Omega$.

2.2. Convergence of domains. For any measurable set $\Omega \in R^{m}$, the characteristic function $\chi_{\Omega} \equiv \chi$ is defined as usual by $\chi(x)=1$ if $x \in \Omega$ and $=0$ if $x \in \Omega^{c}\left(\Omega^{c}=R^{m} \backslash \Omega\right)$. If the Lebesgue measure of $\Omega$ is finite, then $\chi \in L^{1}\left(R^{m}\right)$. Let us consider the gradient $\nabla \chi$ as a distribution in $R^{m}$ defined by

$$
\langle\nabla \chi, \phi\rangle=-\int_{\Omega} \nabla \cdot \phi d x
$$

for any $\phi \in C_{0}^{\infty}\left(R^{m}\right)$. Using the well-known Stokes formula, we get

$$
\langle\nabla \chi, \phi\rangle=-\int_{\Gamma} \phi \cdot n d \Gamma=-\left\langle{ }^{*} \gamma n, \phi\right\rangle,
$$

that is the gradient of $\chi$ takes the form

$$
\nabla \chi=-{ }^{*} \gamma n \text {. }
$$

Here $\Gamma$ denotes the boundary of $\Omega$ and $\gamma$ is the trace operator : $C^{k}(\Omega) \rightarrow C^{k}(\Gamma)$ and ${ }^{*} \gamma$ denotes its transposed operator. Further we have

$$
{ }^{*} \gamma \in\left(C_{0}^{k-1}\left(R^{m}\right)\right)^{\prime},
$$

for any $k \geq 1$. In general $\nabla \chi$ is an element of $\left(C^{k}(\Omega)\right)^{\prime}$. We remark that if $\Omega \in C^{1}$, then $\chi \in \tilde{W}^{s, q}\left(R^{m}\right)$ for $s<1 / q, 1 \leq q<\infty$.

In addition to the above notation we introduce the shape functional

$$
J(\Omega)=\operatorname{meas}(\Gamma)=\int_{\Gamma} d \Gamma,
$$

when the integral makes sense. The functional $J(\Omega)$ can be extended to the following class of non-smooth measurable domains.

A measurable set $E \subset R^{m}$ is said to have the finite perimeter $\mathcal{P}(E)$ provided that $\operatorname{meas}(E)<\infty$ and

$$
\mathcal{P}(E)=\sup _{\phi}\left\{\int_{E} \nabla \cdot \phi d x\left|\phi \in C_{0}^{1}\left(R^{m}\right), \max _{x}\right| \phi(x) \mid<\infty\right\}<\infty .
$$

The class of measurable sets with finite perimeters has been introduced by Caccioppoli. If $E \in C^{2}$, we have

$$
\mathcal{P}(E)=\int_{E} \nabla \cdot n d x=J(\Omega)
$$


Now we introduce the char-topology. Let $D$ be a bounded domain of $R^{3}$. Let

$$
C h(D)=\left\{\chi \in L^{2}(D) \mid \chi(1-\chi)=0 \text { a.e. in } D\right\},
$$

the family of characteristic functions of measurable subsets of $R^{3}$. A char-topology can be defined on the family of measurable subsets of $D$ and in particular for $O(D)$ (open sets of $D$ ) by the $L^{2}$-metric

$$
d_{c h}\left(\Omega_{1}, \Omega_{2}\right)=\int_{D}\left|\chi_{\Omega_{1}}-\chi_{\Omega_{2}}\right| d x
$$

A classical result states that the $L^{2}$-unit ball is weakly compact in the $L^{2}$-topology. This statement does not give the compactness in the space of domains. To obtain a strong compactness result, we consider the measurable sets in $D$ with finite generalized perimeter.

The main result concerning the generalized perimeter is the next one; see [15].

Proposition 2.3. Let $\left\{\Omega_{n}\right\}$ be sequence of measurable sets in D, with $P\left(\Omega_{n}\right) \leq c$. Then there exists a subsequence $\left\{\Omega_{n}\right\}$ and a measurable set $\Omega$ in $D$, such that $\mathcal{P}(\Omega) \leq$ $\lim \inf \mathcal{P}\left(\Omega_{n}\right)$ and $\Omega_{n} \rightarrow \Omega$ in the char-topology.

We note that the generalized perimeter is associated to measurable sets, the charconvergence has not special behavior for the family of open sets where usually p.d.e. are defined. So it is possible that a sequence of open sets with uniformly bounded generalized perimeter converges in the char-topology to measurable set which has not open representative. We denote by $B P S(D)$ the family of finite perimeter sets of $D$.

It is immediate to see that $\left\{\chi_{\Omega} \mid \Omega \in B P S(D)\right\}$ is contained in $B V(D)$ (bounded variation functions in $D$ ). Further $\nabla \chi$ is a Radon measure on $\Omega$ and its total variation $|\nabla \chi|(\Omega)$ is equal to $\mathcal{P}$. The family of finite perimeter sets is important to study the free surface problem in presence of surface tension; see [9].

To consider the free surface or interface problem one needs to introduce a family of perturbations $\Omega_{t}$ of a given domain $\Omega \subset R^{m}$ for $t \geq 0$. It is assumed that the domains $\Omega \equiv \Omega_{0}$ and $\Omega_{t}$ have the same topological properties. Hence one can construct a family of transformations $T_{t}: R^{m} \rightarrow R^{m}$ which are one-to-one, and $T_{t}$ maps $\Omega$ onto $\Omega_{t}$. To construct the transformation $T_{t}$, we consider a vector field $v(x, t)$.

Let

$$
\partial_{t} x=v(x, t), \quad x(y, 0)=y \in \Omega .
$$

The transformation $T_{t}$ is given by

$$
x(t)=T_{t}(y) .
$$

We remark that if $\Omega \subset D\left(\subset R^{m}\right)$ and $v \cdot n=0$ on $\partial D$ we have $T_{t} \bar{\Omega} \subset \bar{D}$. Let $\Gamma(t)$ be the boundary of $\Omega(t)$.

An important result is (see [15])

$$
\begin{aligned}
& d_{t} \int_{\Gamma(t)} d \Gamma=\int_{\Gamma(t)}(\nabla \cdot v(t)-(\nabla v(t) \cdot n, n)) d \Gamma= \\
& \int_{\Gamma(t)}(\nabla \cdot)_{\Gamma} v d \Gamma=\int_{\Gamma(t)} v \cdot n \mathcal{H} d \Gamma .
\end{aligned}
$$


In the above formula we have used the relations

$$
(\nabla \cdot)_{\Gamma}((v \cdot n) n)=(v \cdot n)(\nabla \cdot)_{\Gamma} n
$$

and

$$
(\nabla \cdot)_{\Gamma} n=\mathcal{H}
$$

$\left(\mathcal{H}\right.$ is the mean curvature of $\Gamma$.) Here $(\nabla \cdot)_{\Gamma}$ denotes the tangential divergence to $\Gamma$.

Further we have the following formula for integration by parts on $\Gamma$.

Proposition 2.4 (Integration by parts on $\Gamma$ ). Let $\Omega \in C^{2}$ with boundary $\Gamma$ and $f, \phi \in$ $\tilde{H}^{2}(\Omega)$. Then

$$
\int_{\Gamma} \partial_{x_{i}} f \phi d \Gamma=-\int_{\Gamma} f \partial_{x_{i}} \phi d \Gamma+\int_{\Gamma}\left(\partial_{n}(f \phi)+\mathcal{H} f \phi\right) n_{i} d \Gamma .
$$

Assuming $f=1$ and $\Gamma \in C^{2}$, we can consider $\mathcal{H} n$ as a distribution in $R^{m}$ with support on $\Gamma$, with norm

$$
\|\mathcal{H} n\|_{-s} \leq c(\operatorname{meas}(\Gamma))\|\| \phi \|_{s}
$$

with $s \geq 3$.

The above results permit us to extend the functional vector mean curvature $\mathcal{H} n$ to BPS-sets in $R^{m}$.

2.3. Hyperelastic material. Now we need some results from the elastic theory. $X \in R^{m}$ is the material description of a solid point, also called the Lagrangian coordinate; $x=$ $\psi(X, t)$ is the position of the particle $X$ at time t, the current or final spatial coordinate also called Eulerian coordinate. $x=\psi(X, t))$ is the representation of the deformation of the structure. The velocity $v=\dot{x}$, where the dot indicates the partial derivative with respect to time with $X$ fixed (the material or convective derivative). In the Eulerian description $(x, t)$, the chain rule gives $\dot{\phi}=\phi_{t}+v \cdot \nabla \phi$ for any smooth function $\phi$.

Classical mechanics assumes that $\psi: R^{m} \rightarrow R^{m}$ is a diffeomorphism and the deformation gradient $F=\left[\partial x_{i} / \partial X_{j}\right]=x_{i, j}$ is the Jacobian of the mapping $\psi$ and satisfies

$$
F \in G L_{+}\left(R^{m}\right)=\left\{F \in R^{m \times m} \mid J=\operatorname{det} F>0\right\} .
$$

For incompressible material $J=1$.

The stress tensor referred to the initial configuration of the solid and associated by work with deformation gradient $F$ is denoted $S$ and is usually called the first PiolaKirchhoff stress tensor. We denote by $T$ the actual stress tensor that is referred to the current configuration (Eulerian description) and it is called the true stress or Cauchy stress. The relationship of $T$ and $S$ is the following

$$
T=\left(T_{i j}\right)=(1 / J) S F^{T}=(1 / J) \frac{\partial x_{j}}{\partial X_{k}} S_{i k}
$$

If the elastic part of the stress of a solid particle depends only upon the deformation gradient $F$, it must take the form $\mathcal{D} \mathcal{W}(F)$ where $\mathcal{W}: R^{m \times m} \rightarrow R$ is the strain-energy function and $\mathcal{D}$ is the derivation with respect the arguments of $\mathcal{W}$. Note, however, that $\mathcal{W}$ is not path-independent; it does not represent a potential except if the material is elastic in finite strain. In this case the material is called hyperelastic. In this paper the mathematical investigation of elastic solids allows for large strains. We consider two orders 
tensors as invertible matrices $\mathrm{Mat}^{+}(n)$ and adopt the notation of calculus of matrices. In particular the Frobenius inner product of matrices $A, B$ is denoted $A \cdot B=\sum_{i, j} A_{i j} B_{i j}$ and $|A|^{2}=\operatorname{tr}\left(A A^{T}\right)$.

2.4. Discontinuous model. Let $\Omega$ be a domain of $R^{3}$, with the boundary $\partial \Omega$ and $T$ an arbitrary positive number and $Q_{T}=\Omega \times(0, T)$. We assume that $\Omega$ is filled with a viscous incompressible fluid containing solid particles and write

$$
\Omega=\Omega^{1}(t) \cup \Omega^{2}(t)
$$

for $0 \leq t<T$, where $\Omega^{1}$ is the region occupied by the fluid and $\Omega^{2}$ is the region occupied by solids.

The interface $\Gamma(t)$ between the two phases is expressed by

$$
\Gamma(t)=\left(\partial \Omega^{1}(t) \cup \partial \Omega^{2}(t)\right) \backslash \partial \Omega
$$

for $0 \leq t \leq T$.

Now we recall the basic systems of equations deduced in [13].

Here and in what follows we shall use the well-known notation of vector analysis and summation convention.

We introduce the characteristic functions of $\Omega^{1}(t), \Omega^{2}(t)$, namely $\chi^{1} \equiv \chi(t), \chi^{2}=$ $1-\chi(t)$, respectively.

The interface between the fluid and the solid particles is defined as the discontinuity surface of $\chi \cdot v_{1}, v_{2}$ denote the velocity of the fluid and of a solid point, respectively. Analogously $\left(\rho_{1}, \rho_{2}\right),\left(\mu_{1}, \mu_{2}\right)$ denote the density and the viscosity of the fluid and of the solid, respectively. We introduce $v=\chi^{1} v_{1}+\chi^{2} v_{2}, \rho=\chi^{1} \rho_{1}+\chi^{2} \rho^{2}$ and $\mu=\chi^{1} \mu_{1}+\chi^{2} \mu_{2}$.

Concerning the evolution of $F$, we assume that $F$ is defined in $\Omega$. An application of chain rule gives an Eulerian description of the evolution of deformation gradient $F$,

which we write

$$
d_{t} F=\partial_{t} \frac{\partial x}{\partial X}(X, t)=\frac{\partial v}{\partial X}(x, t)=\frac{\partial v}{\partial x}(x, t) \frac{\partial x}{\partial X}(X, t),
$$

$$
F_{t}+v \cdot \nabla F=(\nabla v) F .
$$

The product on the right is a product of matrices.

The equations of the flow of an incompressible Newtonian fluid carrying incompressible visco-hyperelastic (macroscopic) particles are

$$
\begin{aligned}
& \rho\left(v_{t}+v \cdot \nabla v\right)-\nabla \cdot(\mu \nabla v)+\nabla p-\nabla \cdot\left(\mathcal{D} \mathcal{W}(F) F^{T}\right)=\rho f-\nabla \chi^{2} \mathcal{H}, \\
& \nabla \cdot v=0 \\
& \chi_{t}+v \cdot \nabla \chi=0, \\
& F_{t}+v \cdot \nabla F=(\nabla v) F, \\
& v(x, t)=0, x \in \partial \Omega, v=0 \text { at } \infty, v(x, 0)=v_{0}, F(x, 0)=F_{0}, x \in \Omega, \\
& \chi_{0} \equiv \chi(0)=\text { characteristic function of } \Omega^{1}(0) .
\end{aligned}
$$

We recall that $\rho$ and $\mu$ are positive functions of $\chi$. Of course we need to compute $F$ in the solid. We observe that if we define $F \equiv \chi^{2} F$, thanks to $(2.6)_{3}$, it is a solution of (2.5) with initial data $\chi_{0}^{2} F_{0}$. So far to localize $F$ in the solid part it is sufficient to localize in 
the solid region the initial value $F_{0}$. Without loss of generality we can assume $F=0$ on $\partial \Omega$.

One interesting observation is that the convective derivative of the divergence of $F^{T}$ or $\left(F_{i j, i}\right)$ vanishes if $\nabla \cdot v=0$. In fact if we take the divergence of (2.5) we obtain

$$
\left(F_{i j, i}\right)_{t}+v_{k} F_{i j, i k}+v_{k, i} F_{i j, k}=v_{i, i l} F_{l j}+v_{i, l} F_{l j, i}
$$

or

$$
\partial_{t} \nabla \cdot F^{T}+(v \cdot \nabla) \nabla \cdot F^{T}=0 .
$$

We notice that the material derivative of the divergence of the localization $\chi^{2} F$ vanishes, but $\nabla \cdot\left(\chi^{2} F\right)$ may be a measure. In fact

$$
\nabla \cdot\left(\chi_{0}^{2} F_{0}^{T}\right)=\chi_{0}^{2} \nabla \cdot F_{0}^{T}+\nabla \chi_{0}^{2} F_{0}^{T}=\chi_{0}^{2} \nabla \cdot F_{0}^{T}+n \cdot F_{0}^{T} \delta(\Gamma(0)),
$$

where $\delta(\Gamma(0))$ is Dirac distribution with support on $\Gamma(0)$. Any way we will take advantage of the evolution properties of $F$ for the existence theory in the large without smallness conditions considering the system (2.9) below.

2.5. Continuous model. We assume now that the constituent elastic particles involve molecular scales only. The binary system can be considered as a mixture and one of the volume concentrations $c_{1}(x, t)$ serving as the order parameter. Of course, in this situation, the capillarity effect cannot be defined. Then, as in subsection 4.3 , we define the mean-volume velocity $v=c_{1} u_{1}+c_{2} u_{2}$, the density of mixture $\rho=c_{1} \rho_{1}+c_{2} \rho_{2}$, the viscosity of the mixture $\mu=c_{1} \mu_{1}+c_{2} \mu_{2}$ and assume the stress tensor of the medium is $-(\mu \nabla v)+p I-\mathcal{D} \mathcal{W}(F) F^{T}$. Then the motion of the medium, apart from diffusion process, is governed by the following system (continuous model)

$$
\begin{aligned}
& \rho\left(v_{t}+v \cdot \nabla v\right)-\nabla \cdot(\mu \nabla v)+\nabla p-\nabla \cdot\left(\mathcal{D} \mathcal{W}(F) F^{T}\right)=\rho f, \\
& \nabla \cdot v=0 \\
& c_{t}+v \cdot \nabla c=0 \\
& F_{t}+v \cdot \nabla F=(\nabla v) F \\
& v(x, t)=0, x \in \partial \Omega, v=0 \text { at } \infty, v(x, 0)=v_{0}, \\
& F(x, 0)=F_{0}, c(x, 0)=c_{0}, x \in \Omega .
\end{aligned}
$$

Here $c \equiv c_{1}$, and $c_{1}+c_{2}=1$. In the following instead of (2.7) we will discuss the system

$$
\begin{aligned}
& \rho\left(v_{t}+v \cdot \nabla v\right)-\nabla \cdot(\mu \nabla v)+\nabla p-\nabla \cdot\left(\mathcal{D} \mathcal{W}(F) F^{T}\right)=\rho f, \\
& \nabla \cdot v=0, \\
& \rho_{t}+v \cdot \nabla \rho=0, \quad \mu_{t}+v \cdot \nabla \mu=0 \\
& F_{t}+v \cdot \nabla F=(\nabla v) F, \\
& v(x, t)=0, x \in \partial \Omega, v=0 \text { at } \infty, v(x, 0)=v_{0}, F(x, 0)=F_{0}, \\
& \rho(0)=\rho_{0}, \quad \mu(0)=\mu_{0} .
\end{aligned}
$$

System (2.8) does not change the fundamental structure of (2.7), and it is a bit more general. 
2.6. Small-strain and relaxed models. Equation $(2.6)_{4}$ is responsible of much of the mathematical difficulty to the existence theory of weak solution of (2.6), (2.8). Considerations of analytical tractability of $(2.6)_{4}$ can be derived from small-strain elasticity theory (see [6]). This theory leads to consider the following evolution equation for $F$

$$
F_{t}+v \cdot \nabla F=(W v) F .
$$

Here $W v=\frac{1}{2}\left(\partial_{x_{i}} v^{j}-\partial_{x_{j}} v^{i}\right)$. We call the small strain model the particular model obtained from the complete system (2.6) with the above evolution equation of $F$. Moreover, we remark that this model is also related to a particular Oldroyd-B model (see [13]). Any way, the small-strain condition is a severe restriction for the system solid/fluid considered, i.e. elastic particles transported in a fluid medium. The properties of the evolution equation of $F$ suggest the following relaxed form of (2.6).

$$
\begin{aligned}
& \rho\left(v_{t}+v \cdot \nabla v\right)-\nabla \cdot(\mu \nabla v)+\nabla p-\nabla \cdot\left(\mathcal{D} \mathcal{W}(F) F^{T}\right)=\rho f-\nabla \chi^{2} \mathcal{H}, \\
& \nabla \cdot v=0, \\
& \chi_{t}+v \cdot \nabla \chi=0, \\
& g_{t}+v \cdot \nabla g=0, \\
& \nabla \cdot F^{T}=g \\
& v(x, t)=0, x \in \partial \Omega, v=0 \text { at } \infty, v(x, 0)=v_{0}, g(x, 0)=g_{0}, \\
& \chi_{0} \equiv \chi(0)=\text { characteristic function of } \Omega^{1}(0) .
\end{aligned}
$$

For the small-strain and relaxed models an existence theory of weak solution is developed below.

3. Statement of main results. First, we give the definitions of weak and weak-measure solution of systems (2.6), (2.8), (2.9). Mutatis mutandis, the definitions hold for the smallstrain model. We set $\mathcal{T} \equiv \mathcal{D} \mathcal{W}(F) F^{T}$. For simplicity of exposition we assume supp $\chi_{0}^{2}$ is a bounded open set.

DeFinition 3.1. A weak solution of $(2.6)$ is $(v, F, \chi)$ such that:

i) $\chi \in L^{\infty}\left(Q_{T}\right)$ is a characteristic function,

ii) $v \in L^{2}(0, T ; \tilde{V}(\Omega)) \cap L^{\infty}\left(0, T ; L^{2}(\Omega)\right)$,

iii) $F \in L^{2}\left(Q_{T}\right)$,

iv) for any $T>0$ and any $\phi \in C^{1}\left(0, T ; \mathcal{C}_{0}^{\infty}(\Omega)\right), \phi(T)=0$, and $\eta, \psi \in C^{1}\left(0, T ; C_{0}^{\infty}(\Omega)\right), \eta(T)=\psi(T)=0$,

$$
\begin{aligned}
& \int_{0}^{T}\left(\left(\rho v, \partial_{t} \phi\right)+(\rho(v \cdot \nabla) \phi, v)-(\mu \nabla v+\mathcal{T}, \nabla \phi)+\right. \\
& (\rho f, \phi)-(\mathcal{H}, \phi)) d t+\left(\rho_{0} v_{0}, \phi(0)\right)=0, \\
& \int_{0}^{T}\left(\left(\chi, \eta_{t}\right)+(\chi,(v \cdot \nabla) \eta)\right) d t+\left(\chi_{0}, \eta(0)\right)=0, \\
& \int_{0}^{T}\left(\left(F, \psi_{t}\right)+(F,(v \cdot \nabla) \psi)+(\nabla v F, \psi)\right) d t+\left(F_{0}, \psi(0)\right)=0,
\end{aligned}
$$


with $\mathcal{H} \in L^{\infty}\left(0, T ; \tilde{H}^{-3}(\Omega)\right)$ a functional which represents the generalized vector mean curvature.

Definition 3.2. A weak solution of $(2.8)$ is $(v, \rho, \mu, F)$ such that:

i) $\rho, \mu \in L^{\infty}\left(Q_{T}\right)$,

ii) $v \in L^{2}(0, T ; \tilde{V}(\Omega)) \cap L^{\infty}\left(0, T ; L^{2}(\Omega)\right)$,

iii) $F \in L^{2}\left(Q_{T}\right)$,

iv) for any $T>0$ and any $\phi \in C^{1}\left(0, T ; \mathcal{C}_{0}^{\infty}(\Omega)\right), \phi(T)=0$, and $\eta, \psi \in C^{1}\left(0, T ; C_{0}^{\infty}(\Omega)\right), \eta(T)=\psi(T)=0$,

$$
\begin{aligned}
& \int_{0}^{T}\left(\left(\rho v, \partial_{t} \phi\right)+(\rho(v \cdot \nabla) \phi, v)-(\mu \nabla v+\mathcal{T}, \nabla \phi)+(\rho f, \phi)\right) d t+\left(\rho_{0} v_{0}, \phi(0)\right)=0, \\
& \int_{0}^{T}\left(\left(\rho, \eta_{t}\right)+(\rho,(v \cdot \nabla) \eta)\right) d t+\left(\rho_{0}, \eta(0)\right)=0, \\
& \int_{0}^{T}\left(\left(\mu, \eta_{t}\right)+(\mu,(v \cdot \nabla) \eta)\right) d t+\left(\mu_{0}, \eta(0)\right)=0, \\
& \int_{0}^{T}\left(\left(F, \psi_{t}\right)+(F,(v \cdot \nabla) \psi)+(\nabla v F, \psi)\right) d t+\left(F_{0}, \psi(0)\right)=0 .
\end{aligned}
$$

Definition 3.3. A weak solution of $(2.9)$ is $(v, \chi, g, F)$ such that:

i) $\chi \in L^{\infty}\left(Q_{T}\right)$ is a characteristic function,

ii) $v \in L^{2}(0, T ; \tilde{V}(\Omega)) \cap L^{\infty}\left(0, T ; L^{2}(\Omega)\right)$,

iii) $g \in L^{\infty}\left(Q_{T}\right), F \in L^{\infty}\left(0, T ; H_{0}^{1}(\Omega)\right)$,

iv) for any $T>0$ and any $\phi \in C^{1}\left(0, T ; \mathcal{C}_{0}^{\infty}(\Omega)\right), \phi(T)=0$, and $\eta, \psi \in C^{1}\left(0, T ; C_{0}^{\infty}(\Omega)\right), \eta(T)=\psi(T)=0$,

$$
\begin{aligned}
& \int_{0}^{T}\left(\left(\rho v, \partial_{t} \phi\right)+(\rho(v \cdot \nabla) \phi, v)-(\mu \nabla v+\mathcal{T}, \nabla \phi)+\right. \\
& (\rho f, \phi)-(\mathcal{H}, \phi)) d t+\left(\rho_{0} v_{0}, \phi(0)\right)=0 \\
& \int_{0}^{T}\left(\left(\chi, \eta_{t}\right)+(\chi,(v \cdot \nabla) \eta)\right) d t+\left(\chi_{0}, \eta(0)\right)=0, \\
& \int_{0}^{T}\left(\left(g, \psi_{t}\right)+(g,(v \cdot \nabla) \psi)\right) d t+\left(g_{0}, \psi(0)\right)=0, \\
& \nabla \cdot F^{T}=g \text { a.e. in } Q_{T},
\end{aligned}
$$

with $\mathcal{H} \in L^{\infty}\left(0, T ; \tilde{H}^{-3}(\Omega)\right)$ a functional which represents the generalized vector mean curvature.

DeFinition 3.4. A weak-measure solution of $(2.6)$ is $(v, F, \Lambda, \chi)$ such that:

i) $\quad \chi \in L^{\infty}\left(Q_{T}\right)$ is a characteristic function,

ii) $\Lambda$ a Radon measure,

iii) $v \in L^{2}(0, T ; \tilde{V}(\Omega)) \cap L^{\infty}\left(0, T ; L^{2}\left(Q_{T}\right)\right)$,

iv) $F \in L^{2}\left(Q_{T}\right), \quad \nabla \cdot F^{T} \in L^{\infty}\left(Q_{T}\right)$,

v) for any $\phi \in C^{1}\left(0, T ; \mathcal{C}_{0}^{\infty}(\Omega)\right), \eta, \psi, \xi \in C^{1}\left(0, T ; C_{0}^{\infty}(\Omega)\right)$, 


$$
\begin{aligned}
\phi(T)= & \eta(T)=\psi(T)=\xi(T)=0, \\
& \int_{0}^{T}\left(\left(\rho v, \partial_{t} \phi\right)+((\rho v \cdot \nabla) \phi, v)-(\mu \nabla v+\Lambda, \nabla \phi)+\right. \\
& (\rho f, \phi)-(\mathcal{H}, \phi)) d t+\left(\rho_{0} v_{0}, \phi(0)\right)=0, \\
& \int_{0}^{T}\left(\left(\chi, \eta_{t}\right)+(\chi,(v \cdot \nabla) \eta)\right) d t+\left(\chi_{0}, \eta(0)\right)=0, \\
& \int_{0}^{T}\left(\left(F, \psi_{t}\right)+(F,(v \cdot \nabla) \psi)-\left(v \nabla \cdot F^{T}, \psi\right)-(F, \nabla \psi v)\right) d t+\left(F_{0}, \psi(0)\right)=0, \\
& \int_{0}^{T}\left(\left(\nabla \cdot F^{T}, \xi_{t}\right)+\left(\nabla \cdot F^{T},(v \cdot \nabla) \xi\right)\right) d t+\left(\nabla \cdot F_{0}^{T}, \xi(0)\right)=0,
\end{aligned}
$$

with $\mathcal{H} \in L^{\infty}\left(0, T ; \tilde{H}^{-3}(\Omega)\right)$ a functional which represents the generalized vector mean curvature.

Definition 3.5. A weak-measure solution of $(2.8)$ is $(v, F, \Lambda, \rho, \mu)$ such that:

i) $\rho, \mu \in L^{\infty}\left(Q_{T}\right)$,

ii) $\Lambda$ a Radon measure,

iii) $v \in L^{2}(0, T ; \tilde{V}(\Omega)) \cap L^{\infty}\left(0, T ; L^{2}\left(Q_{T}\right)\right)$,

iv) $F \in L^{2}\left(Q_{T}\right), \quad \nabla \cdot F^{T} \in L^{\infty}\left(Q_{T}\right)$,

v) for any $\phi \in C^{1}\left(0, T ; \mathcal{C}_{0}^{\infty}(\Omega)\right), \eta, \psi, \xi \in C^{1}\left(0, T ; C_{0}^{\infty}(\Omega)\right)$,

$\phi(T)=\eta(T)=\psi(T)=\xi(T)=0$,

$$
\begin{aligned}
& \int_{0}^{T}\left(\left(\rho v, \partial_{t} \phi\right)+((\rho v \cdot \nabla) \phi, v)-(\mu \nabla v+\Lambda, \nabla \phi)+(\rho f, \phi)\right) d t+\left(\rho_{0} v_{0}, \phi(0)\right)=0, \\
& \int_{0}^{T}\left(\left(\rho, \eta_{t}\right)+(\rho,(v \cdot \nabla) \eta)\right) d t+\left(\rho_{0}, \eta(0)\right)=0, \\
& \int_{0}^{T}\left(\left(\mu, \eta_{t}\right)+(\mu,(v \cdot \nabla) \eta)\right) d t+\left(\mu_{0}, \eta(0)\right)=0, \\
& \int_{0}^{T}\left(\left(F, \psi_{t}\right)+(F,(v \cdot \nabla) \psi)-\left(v \nabla \cdot F^{T}, \psi\right)-(F, \nabla \psi v)\right) d t+\left(F_{0}, \psi(0)\right)=0, \\
& \int_{0}^{T}\left(\left(\nabla \cdot F^{T}, \xi_{t}\right)+\left(\nabla \cdot F^{T},(v \cdot \nabla) \xi\right)\right) d t+\left(\nabla \cdot F_{0}^{T}, \xi(0)\right)=0 .
\end{aligned}
$$

We recall that the above definitions hold if $\operatorname{supp}^{2}(x, 0)$ is a bounded set. If it is unbounded the above definitions continue to hold in local sense, i.e. in the spaces $V_{l o c}(\Omega), L_{l o c}^{2}(\Omega)$ and so on.

Now we present the main results of the paper. We state the theorems in the framework of Sobolev spaces. The first four theorems are devoted to the existence of continuous model, i.e., the density and the viscosity are regular functions or constants. In Theorems $3.10,3.11,3.12$, the existence of weak solution of the continuous and discontinuous model is treated. In the following $\alpha, \beta$ are positive constants such that $\alpha, \beta<1$.

THEOREM 3.6. Let $\Omega$ be an unbounded domain in $R^{3}$ with boundary $\partial \Omega$ uniformly of class $C^{3}$ and $\mathcal{W} \in C^{3}\left(R^{3 \times 3}\right)$. Let $v_{0} \in H^{2}(\Omega) \cap \tilde{V},\left(\nabla \mu_{0}, \nabla \rho_{0}, \nabla F_{0}\right) \in \tilde{H}^{1}(\Omega), F_{0} \in$ 
$L^{\infty}(\Omega) \cap L^{2}(\Omega)$ with $\alpha \leq \rho_{0} \leq 1, \beta \leq \mu_{0} \leq 1$ such that $\left\|\left|u_{0}\right|\right\|_{2}+\left|\left\|\nabla \rho_{0}\left|\left\|_{1}+\left|\left\|\nabla \mu_{0} \mid\right\|_{1}+\right.\right.\right.\right.\right.$ $\left\|\mid F_{0}\right\|_{2} \leq r_{0}, r_{0}$ being given, $f \in L^{2}\left(0, T ; \tilde{H}^{1}(\Omega)\right), \partial_{t} f \in L^{2}\left(\Omega_{T}\right)$.

Then there exists a $\bar{T}\left(r_{0}\right)>0, \bar{T} \leq T$, such that there exists a solution $(v, \rho, \mu, F)$ of (2.8) on $(0, \bar{T})$ such that

$$
\begin{gathered}
v \in L^{2}\left(0, \bar{T} ; H^{3}(\Omega) \cap \tilde{V}\right), \quad \partial_{t} v \in L^{2}(0, \bar{T} ; \tilde{V}), \\
0<\alpha \leq \rho \leq 1, \quad 0<\beta \leq \mu \leq 1, \quad(\rho, \mu, F) \in L^{\infty}\left(Q_{T}\right), \\
(\nabla \rho, \nabla \mu, \nabla F) \in L^{\infty}\left(0, \bar{T} ; \tilde{H}^{1}(\Omega)\right), \quad\left(\partial_{t} \rho, \partial_{t} \mu, \partial_{t} F\right) \in L^{2}\left(0, \bar{T} ; \tilde{H}^{1}(\Omega)\right) .
\end{gathered}
$$

Moreover, the solution $(v, \rho, \mu, F)$ is unique.

THEOREM 3.7. Let $\Omega$ be an unbounded domain in $R^{3}$ with boundary $\partial \Omega$ uniformly of class $C^{3}, q>3$ and $\mathcal{W} \in C^{2}\left(R^{3 \times 3}\right)$. Let $v_{0} \in H^{2}(\Omega) \cap \tilde{V},\left(\rho_{0}, \mu_{0}, F_{0}\right) \in H^{1, q}(\Omega)$ with $0<\alpha \leq \rho_{0} \leq 1,0<\beta \leq \mu_{0} \leq 1$ and $F_{0} \in L^{\infty}(\Omega) \cap L^{2}(\Omega)$ such that $\left\|\left|v_{0}\right|\right\|_{2}+\left|\nabla \rho_{0}\right|_{q}+$ $\left|\nabla \mu_{0}\right|_{q}+\left.||\left|F_{0}\right|\right|_{1, q} \leq r_{0}, r_{0}$ being given, $f \in L^{2}\left(0, T ; \tilde{H}^{1}(\Omega)\right), \partial_{t} f \in L^{2}\left(\Omega_{T}\right)$.

Then there exists a $\bar{T}\left(r_{0}\right)>0, \bar{T} \leq T$, such that there exists a solution $(v, \rho, \mu, F)$ of (2.8) on $(0, \bar{T})$ such that

$$
\begin{gathered}
v \in L^{2}\left(0, \bar{T} ; H^{2, q}(\Omega) \cap \tilde{V}\right), \quad \partial_{t} v \in L^{2}(0, \bar{T} ; \tilde{V}), \\
0<\alpha \leq \rho \leq 1, \quad 0<\beta \leq \mu \leq 1, \quad F \in L^{\infty}\left(Q_{\bar{T}}\right), \\
(\rho, \mu, F) \in L^{\infty}\left(0, \bar{T} ; H^{1, q}(\Omega)\right), \quad\left(\partial_{t} \rho, \partial_{t} \mu, \partial_{t} F\right) \in L^{2}\left(Q_{\bar{T}}\right) .
\end{gathered}
$$

Moreover, the solution $(v, \rho, \mu, F)$ is unique.

REMARK. Theorem 3.7 continues to hold (except for the uniqueness) in the case when $\rho \in L^{\infty}\left(Q_{T}\right)$ only.

THEOREM 3.8. The assumptions of Theorem 3.6 hold with $0 \leq \rho_{0} \leq 1$. Further we assume that $\frac{\nabla \cdot\left(\mu_{0} \nabla v_{0}\right)+\nabla \cdot \mathcal{T}(0)}{\sqrt{\rho_{0}}}$ belongs in $L^{2}(\Omega)$.

Then there exists a $\bar{T}$ such that there exists a solution $(v, \rho, \mu, F)$ of (2.8) satisfying $v \in L^{2}\left(0, \bar{T} ; H^{3}(\Omega) \cap V\right), \quad \sqrt{\rho} v \in L^{\infty}\left(0, \bar{T} ; L^{2}(\Omega)\right), \quad \sqrt{\rho} \partial_{t} v \in L^{\infty}\left(0, \bar{T} ; L^{2}(\Omega)\right)$, $\partial_{t} v \in L^{2}(0, \bar{T} ; V(\Omega)), \quad 0 \leq \rho \leq 1, \quad \beta \leq \mu \leq 1, F \in L^{\infty}\left(Q_{\bar{T}}\right)$, $(\nabla \rho, \nabla \mu, \nabla F) \in L^{\infty}\left(0, \bar{T} ; \tilde{H}^{1}(\Omega)\right), \quad\left(\partial_{t} \rho, \partial_{t} \mu, \partial_{t} F\right) \in L^{2}\left(0, \bar{T} ; \tilde{H}^{1}(\Omega)\right)$.

Moreover, the solution $(v, \rho, \mu, F)$ is unique.

We notice that in Theorem 3.8 we prove the existence of strong solution even though the initial density vanishes in an open subset of $\Omega$, i.e., an initial vacuum is allowed. For the classical nonhomogeneous incompressible fluids the problem of the existence of weak solution with initial vacuum was solved completely in [11]. Higher regularity of solutions in the case of vacuum was solved in the more general context of compressible fluid in [14].

Now we state global results.

Theorem 3.9. Let $\Omega, \mathcal{W}>0, v_{0}, \rho_{0}, \mu_{0}, F_{0}, q$ be as in Theorem 3.7. Besides, assume that $f \in L^{2}\left(0, \infty ; \tilde{H}^{1}(\Omega)\right), \partial_{t} f \in L^{2}\left(0, \infty ; L^{2}(\Omega)\right)$ and $r_{0}+\int_{0}^{\infty}\left(\left\|\left.|| f\left|\|_{1}^{2}+\right| \partial_{t} f\right|_{2} ^{2}\right) d t\right.$ is sufficiently small. Then there exists a (unique) solution $(v, \rho, \mu, F)$ of the problem (2.8) on $(0, T)(T$ is an arbitrary positive number) such that

$$
\left.v \in L^{2}(0, T) ; H^{2, q}(\Omega) \cap \tilde{V}\right), \quad \partial_{t} v \in L^{2}(0, T ; \tilde{V}(\Omega)) \cap L^{\infty}\left(0, T ; L^{2}(\Omega)\right),
$$




$$
\begin{gathered}
0<\alpha \leq \rho \leq 1, \quad 0<\beta \leq \mu \leq 1, \quad F \in L^{\infty}\left(Q_{T}\right), \\
(\nabla \rho, \nabla \mu, \nabla F) \in L^{\infty}\left(0, T ; L^{q}(\Omega)\right), \quad\left(\partial_{t} \rho, \partial_{t} \mu, \partial_{t} F\right) \in L^{2}\left(0, T ; L^{2}(\Omega)\right) .
\end{gathered}
$$

Moreover, the solution $(v, \rho, \mu, F)$ is unique. Further, if $\mathcal{T}=e^{-\lambda t} \mathcal{D} \mathcal{W}(F) F^{T}, \lambda>0$ (decay model), and the Poincaré-Friedrichs inequality holds then $T=\infty$.

The proof of Theorem 3.9 uses the same arguments of Theorem 5.9 in [13] so we omit details.

In the following theorems the existence of weak-measure solutions is considered.

THEOREM 3.10. Let $\chi_{0}$ be the characteristic function of $\Omega^{1}(0), f \in L^{2}\left(Q_{T}\right), F_{0} \in$ $C_{0}^{1}(\Omega) \cap L^{\infty}(\Omega)$ with $n \cdot F_{0}=0$ on $\Gamma(0), v_{0} \in H$, and $a\left(|F|^{2}-1\right)^{+} \leq \mathcal{W} \leq a\left(|F|^{2}+1\right) \quad(a$ is a positive constant). Then there exists a weak-measure solution of (2.6).

We notice that the condition $F_{0} \in C^{1}$ is redundant, but this avoids approximation arguments. Moreover $n \cdot F_{0}=0$ on $\Gamma(0)$ circumvents $\nabla \cdot F^{T}$ to be a measure.

An analogous statement holds for system (2.8) so we omit it.

We notice that Theorem 3.10 is a guidance for the next theorems.

Theorem 3.11. Let $\chi_{0}, v_{0}, f$ be as in Theorem 3.10, $F_{0} \in L^{\infty}(\Omega)$ and $\mathcal{W} \in C^{1}\left(R^{3 \times 3}\right)$. Then there exists a weak solution of small-strain model.

Finally we state the existence of a weak solution for the relaxed model.

TheOREM 3.12. Let $\chi_{0}$ be the characteristic function of $\Omega^{1}(0), \Omega, \mathcal{W}$ as in Theorem 3.11. Moreover, assume that $v_{0} \in H, g_{0} \in L^{\infty}(\Omega) \cap L^{2}(\Omega)$. Then there exists a weak solution $(v, g, F, \chi)$ to (2.9).

We notice, denoting by $E$ the support of $\chi^{2}$ and thanks to the results of De Giorgi, for weak solutions we find the interface $\Gamma(t)$ as the points $(x, t) \in \operatorname{supp}\left|\nabla \chi^{2}\right| \cap \Omega$. Further $\Gamma(t)$ contains, locally, a subset $\Gamma^{*}(t)$ such that there exists a generalized inner normal $n_{E}$ : $\Gamma^{*}(t) \rightarrow \mathbf{S}^{2}$. By the Besicovitch derivation theorem the measure $\left|\nabla \chi^{2}\right|$ is concentrated on $\Gamma^{*}(t)$, and $\nabla \chi^{2}=n_{E}\left|\nabla \chi^{2}\right|$. Moreover $\Gamma^{*}(t)$ is, locally, a countable (2)-rectifiable set. In other words for weak solution we obtain an interface with the classical properties. Indeed the only difference is that the inner normal and the interface are understood in a measure theoretic sense and non in topological one. Moreover, the vector mean curvature can be defined as a functional in $\tilde{H}^{-3}(\Omega)$.

Furthermore, as the weak solution is conceived, the assumption $g \in L^{2}(\Omega)$ is not strictly necessary.

4. Existence theory of the continuous model. In this section we prove the existence theorems for the continuous model via a generalized Lax-Milgram lemma and a Schauder fixed point theorem.

4.1. Proof of Theorem 3.6. First we consider the following auxiliary problem. Let

$$
\mathcal{F}=\left\{\phi \mid \phi \in L^{2}\left(0, T ; H^{2}(\Omega) \cap \tilde{V}\right), \partial_{t} \phi \in L^{2}\left(Q_{T}\right), \text { with the natural norm }\right\},
$$

and let $\Phi$ be the closure of the space

$$
\left\{\phi \mid \phi \in L^{2}\left(0, T ; H^{2}(\Omega) \cap \tilde{V}\right), \partial_{t} \phi \in C_{0}\left(0, T ; H^{2}(\Omega) \cap \tilde{V}\right)\right\}
$$


in the norm

$$
\|\phi\|_{\Phi}=\|\phi\|_{\mathcal{F}}+\|\phi \phi(0)\|_{1} .
$$

We consider the following problem:

find $(v, \rho, \mu, F)$ that satisfies

$$
\begin{aligned}
& \left(\rho \partial_{t} v-\Delta v+k v+\nabla p\right)=-\rho u \cdot \nabla u+\rho f+\nabla \cdot \mathcal{T}-\nabla \cdot((1-\mu) \nabla u)+k u, \\
& \nabla \cdot v=0, \\
& \partial_{t} \rho+u \cdot \nabla \rho=0, \\
& \partial_{t} \mu+u \cdot \nabla \mu=0, \\
& \partial_{t} F+u \cdot \nabla F=\nabla u F, \\
& v(0)=v_{0}, \rho(0)=\rho_{0}, \mu(0)=\mu_{0}, F(0)=F_{0},
\end{aligned}
$$

such that $v \in \mathcal{F},(\nabla \rho, \nabla \mu, \nabla F) \in L^{\infty}\left(0, T ; \tilde{H}^{1}(\Omega)\right),\left(\partial_{t} \rho, \partial_{t} \mu, \partial_{t} F\right) \in L^{\infty}\left(0, T ; \tilde{H}^{1, q}(\Omega)\right)$, $0<\alpha \leq \rho \leq 1,0<\beta \leq \mu \leq 1, F \in L^{\infty}\left(Q_{T}\right) \cap L^{2}\left(Q_{T}\right)$.

Here $u \in L^{2}\left(0, T ; H^{3}(\Omega) \cap \tilde{V}\right), \partial_{t} u \in L^{2}\left(0, T ; H^{1}(\Omega) \cap \tilde{V}\right), v_{0} \in H^{2}(\Omega) \cap \tilde{V}$, $\left.\left.\left(\nabla \rho_{0}, \nabla \mu_{0}, \nabla F_{0}\right) \in \tilde{H}^{1, q}(\Omega)\right), \quad F_{0} \in L^{2}(\Omega) \cap L^{\infty}(\Omega)\right), \alpha \leq \rho \leq 1, \beta \leq \mu \leq 1$ are given functions and $q \geq 2$.

Now, let $y(\tau, x, t)$ denote the position at time $\tau$ of the fluid particle which occupies the position $x$ at time $t$ and it is the solution of

$$
\frac{d y}{d \tau}=u(y, \tau), \quad y(t, x, t)=x
$$

then, the explicit form of $\rho$ is

$$
\rho(x, t)=\rho_{0}(y(0, x, t))
$$

From (4.2) it follows

$$
0<\alpha \leq \rho(x, t) \leq 1
$$

Now applying the gradient operator $\nabla$ to $(4.1)_{3}$, multiplying the result by $|\nabla \rho|^{q-2} \nabla \rho$ and, after integrations, we get

$$
\left.|\nabla \rho|_{q} \leq|\nabla \rho(0)|_{q} \exp \left(c \int_{0}^{t}|\nabla u|_{\infty}\right) d \tau\right)
$$

Analogously we obtain

$$
\|\rho\|_{2}^{2} \leq \exp \left(c \int_{0}^{t}\left(|\nabla u|_{\infty}+1\right) d \tau\right)\left(\left\|\rho_{0}\right\|_{2}^{2}+\int_{0}^{t}|\nabla \rho|_{3}^{2}|\|u\||_{3}^{2} d \tau\right) .
$$

Analogously for $F$ we obtain

$$
\left.0<\left|F_{0}\right| \exp \left(-\int_{0}^{t}|\nabla u|_{\infty}\right) d \tau\right) \leq|F(x, t)| \leq\left|F_{0}\right| \exp \left(\int_{0}^{t}|\nabla u|_{\infty} d \tau\right)
$$

where $|F|^{2}=\operatorname{tr}\left(F F^{T}\right)$,

$$
\left.|\nabla F|_{q} \leq \exp \left(c \int_{0}^{t}|\nabla u|_{\infty}\right) d \tau\right)\left(|\nabla F(0)|_{q}+\int_{0}^{t}|F|_{\infty}|||\nabla u| \|_{1, q} d \tau\right) .
$$


Analogously

$$
\|F\|_{2}^{2} \leq \exp \left(c \int_{0}^{t}\left(|\nabla u|_{\infty}+1\right) d \tau\right)\left(\left\|F_{0}\right\|_{2}^{2}+\int_{0}^{t}\left(|F|_{\infty}^{2}+|\nabla F|_{3}^{2}\right)\||u|\|_{3}^{2} d \tau\right) .
$$

Now we prove the existence of a solution of $(4.1)_{1}$. We let

$$
\begin{aligned}
& E(v, \phi)= \int_{0}^{T}\left(\rho \partial_{t} v+A v+k v, \partial_{t} \phi+A \phi+\phi\right) d t+ \\
&(k v(0), A \phi(0)+\phi(0)), \\
& L(\phi)= \int_{0}^{T}(-\rho u \cdot \nabla u+(\mu-1) \Delta u+\nabla \mu \nabla u+k u+\nabla \cdot \mathcal{T}+ \\
&\left.\rho f, \partial_{t} \phi+A \phi+\phi\right) d t+\left(k v_{0}, A \phi(0)+\phi(0)\right) .
\end{aligned}
$$

Here $A=-P \Delta$ is the Stokes operator and $k$ a suitable positive constant. First, $L(\phi)$ is a linear continuous form on $\Phi$ with respect to the norm $\|\phi\|_{\Phi}$. Moreover, bearing in mind that $|A \phi|_{2}+|\phi|_{2} \geq\left. c|||\phi|\right|_{2}$, we have

$$
\begin{aligned}
& E(\phi, \phi)= \\
& \int_{0}^{T}\left(\left|\sqrt{\rho} \partial_{t} \phi\right|_{2}^{2}+|A \phi|_{2}^{2}+(k+1)|\nabla \phi|_{2}^{2}+k|\phi|_{2}^{2}+\left(\rho \partial_{t} \phi, A \phi+\phi\right) d t+\right. \\
& \frac{1}{2}\left(|\nabla \phi(T)|_{2}^{2}-|\nabla \phi(0)|_{2}^{2}\right)+\frac{k}{2}\left(|\phi(T)|_{2}^{2}-|\phi(0)|_{2}^{2}\right)+k\left(|\nabla \phi(0)|_{2}^{2}+|\phi(0)|_{2}^{2}\right) \geq \\
& \int_{0}^{T}\left(\left|\sqrt{\rho} \partial_{t} \phi\right|_{2}^{2}+|A \phi|_{2}^{2}-\frac{3}{4}\left|\sqrt{\rho} \partial_{t} \phi\right|_{2}^{2}-\frac{1}{2}|A \phi|_{2}^{2}+\left(k-\frac{3}{4}\right)|\phi|_{2}^{2}\right) d t+\frac{1}{2}\left(|\nabla \phi(T)|_{2}^{2}+\right. \\
& \left.|\nabla \phi(0)|_{2}^{2}\right)+\frac{1}{2}\left(|\phi(T)|_{2}^{2}+|\phi(0)|_{2}^{2}\right) \geq c\|\phi\|_{\Phi}^{2} .
\end{aligned}
$$

Then, thanks to the Lax-Milgram theorem, there exists a $v \in \mathcal{F}$ such that

$$
E(v, \phi)=L(\phi)
$$

is satisfied for every $\phi \in \Phi$.

Now let $\tilde{\phi}$ be a solution of the problem

$$
\begin{aligned}
& \partial_{t} \tilde{\phi}+A \tilde{\phi}+\tilde{\phi}=0, \\
& \tilde{\phi}(0)=h(x), \\
& \nabla \cdot \tilde{\phi}=0, \tilde{\phi}=0, \text { on } \partial \Omega \text { and at } \infty
\end{aligned}
$$

with $h(x)$ smooth enough and divergence free.

Replacing in (4.9) $\phi$ with $\tilde{\phi}$ we have

$$
(v(0), A h+h)=\left(v_{0}, A h+h\right),
$$

which implies $v(0)=v_{0}$.

Now let $\bar{\phi}$ be a solution of the problem

$$
\begin{aligned}
& \partial_{t} \bar{\phi}+A \bar{\phi}+\bar{\phi}=g(x, t), \\
& \bar{\phi}(0)=0, \\
& \nabla \cdot \bar{\phi}=0, \bar{\phi}=0, \text { on } \partial \Omega \text { and at } \infty,
\end{aligned}
$$


with $g$ smooth enough and solenoidal. Replacing $\phi$ with $\bar{\phi}$ in (4.9), we have

$$
\int_{0}^{T}\left(\rho \partial_{t} v+\rho u \cdot \nabla u-\nabla \cdot \mathcal{T}-\rho f-\Delta v+k(v-u)-\nabla \cdot((\mu-1) \nabla u), g\right) d t=0 .
$$

This implies that $(v, \rho, \mu, F)$ satisfies a.e. in $Q_{T}$ the following system:

$$
\begin{aligned}
& \rho \partial_{t} v+\rho u \cdot \nabla u-\nabla \cdot \mathcal{T}+\nabla p-\Delta v+k(v-u)-\rho f- \\
& \nabla \cdot((\mu-1) \nabla u)=0, \\
& \partial_{t} \rho+\nabla \cdot(\rho u)=0, \\
& \partial_{t} \mu+\nabla \cdot(\mu u)=0, \\
& \partial_{t} F+u \cdot \nabla F=\nabla u F .
\end{aligned}
$$

with $v(0)=v_{0}, \rho(0)=\rho_{0}, \mu(0)=\mu_{0}, F(0)=F_{0}$.

Here $p$ is the pressure defined in classical manner. Now we prove more regularity for $v$. To avoid tedious calculations and notation, we work directly with the derivatives with respect to $t$ of $v$ instead of the difference quotients.

First, we multiply $(4.10)_{1}$ by $v$, and integrate over $Q_{t}$, to obtain

$$
\begin{aligned}
|\sqrt{\rho} v|_{2}^{2}+ & \int_{0}^{t}\left(k|v|_{2}^{2}+|\nabla v|_{2}^{2}\right) d \tau \leq|\sqrt{\rho}(0) v(0)|_{2}^{2}+\delta \int_{0}^{t}|\nabla v|^{2} d \tau+ \\
c_{\delta} & {\left[\int_{0}^{t}\left(|u|_{\infty}^{2}|\sqrt{\rho} v|_{2}^{2}+|u|_{6}^{2}|\nabla u|_{3}^{2}+k|u|_{2}^{2}+|(\mu-1) \nabla u|_{2}^{2}+|\mathcal{T}|_{2}^{2}+|f|_{2}^{2}\right) d \tau\right] . }
\end{aligned}
$$

We multiply $(4.10)_{1}$ by $\partial_{t} v$, integrate over $Q_{t}$, obtain

$$
\begin{aligned}
& \frac{2}{3} \int_{0}^{t}\left|\sqrt{\rho} \partial_{\tau} v\right|_{2}^{2} d \tau+\frac{k}{2}|v(t)|_{2}^{2}+\frac{1}{2}|\nabla v(t)|_{2}^{2} \leq \frac{1}{2}\left(|\nabla v(0)|_{2}^{2}+k|v(0)|_{2}^{2}\right)+ \\
& c_{\delta}\left[\int_{0}^{t}\left(|u|_{6}^{2}|\nabla u|_{3}^{2}+|(\mu-1) \nabla u|_{2}^{2}+k|u|_{2}^{2}+|\mathcal{T}|_{2}^{2}+|f|_{2}^{2}\right) d \tau\right]+ \\
& \delta \int_{0}^{t}\left(\left|\partial_{\tau} \nabla v\right|^{2}+\left|\partial_{\tau} v\right|^{2}\right) d \tau
\end{aligned}
$$

Now we differentiate $(4.10)_{1}$ with respect to $t$ and obtain

$$
\begin{aligned}
& \rho \partial_{t}^{2} v+\partial_{t} \rho \partial_{t} v+k \partial_{t} v+\partial_{t} \rho u \cdot \nabla u+\rho \partial_{t} u \cdot \nabla u+\rho u \cdot \nabla \partial_{t} u- \\
& \nabla \cdot \partial_{t} \mathcal{T}-\nabla \cdot\left((\mu-1) \nabla \partial_{t} u\right)-\nabla \cdot\left(\partial_{t} \mu \nabla u\right)-k \partial_{t} u- \\
& \partial_{t} \rho f-\rho \partial_{t} f-\Delta \partial_{t} v+\nabla \partial_{t} p=0 .
\end{aligned}
$$

Multiplying (4.13) by $\partial_{t} v$, integrating over $Q_{t}$ and bearing in mind (2.1), we find

$$
\begin{aligned}
& \frac{1}{2}\left|\sqrt{\rho}(t) \partial_{t} v(t)\right|_{2}^{2}+\frac{1}{2} \int_{0}^{t}\left(\left|\nabla \partial_{\tau} v\right|_{2}^{2}+k\left|\partial_{t} v\right|_{2}^{2}\right) d \tau \leq \frac{1}{2}\left|\sqrt{\rho}(0) \partial_{t} v(0)\right|_{2}^{2}+ \\
& c_{\delta}\left[\int _ { 0 } ^ { t } \left(|\sqrt{\rho} u|_{\infty}^{2}\left|\sqrt{\rho} \partial_{\tau} v\right|_{2}^{2}+|\nabla u|_{2}^{4}\left|D^{2} u\right|_{2}^{2}+|\nabla u|_{2}^{6}+\left|\sqrt{\rho} \partial_{\tau} u\right|_{2}^{2}|\nabla u|_{3}^{2}+\right.\right. \\
& \left|\partial_{t} \mathcal{T}\right|_{2}^{2}+\left|\partial_{\tau} \mu \nabla u\right|_{2}^{2}+k\left|\partial_{t} u\right|_{2}^{2}+\left|\partial_{\tau} f\right|_{2}^{2}+\left|\partial_{\tau} \rho\right|_{2}^{2}|f|_{3}^{2}+ \\
& \left.\left.\left|(\mu-1) \nabla \partial_{\tau} u\right|_{2}^{2}\right) d \tau\right]+\delta \int_{0}^{t}\left|\nabla \partial_{\tau} v\right|_{2}^{2} d \tau
\end{aligned}
$$


Now we sum (4.11), (4.12) and (4.14), and for suitable $\delta$, we obtain

$$
\begin{aligned}
& \frac{1}{2}\left|\sqrt{\rho}(t) \partial_{t} v(t)\right|_{2}^{2}+\frac{1}{2}\left(|\nabla v(t)|_{2}^{2}+k|v(t)|_{2}^{2}\right)+\frac{1}{3} \int_{0}^{t}\left(\left|\sqrt{\rho}(t) \partial_{\tau} v(t)\right|_{2}^{2}+\right. \\
& \left.k\left|\partial_{t} v\right|_{2}^{2}+\left|\nabla \partial_{\tau} v\right|_{2}^{2}\right) d \tau \leq \frac{1}{2}\left|\sqrt{\rho}(0) \partial_{t} v(0)\right|_{2}^{2}+\frac{1}{2}|\nabla v(0)|_{2}^{2}+ \\
& c_{\delta}\left[\int _ { 0 } ^ { t } \left(|u|_{\infty}^{2}\left|\sqrt{\rho} \partial_{t} v\right|_{2}^{2}+|\nabla u|_{2}^{4}\left|D^{2} u\right|_{2}^{2}+|\nabla u|_{2}^{6}+\left|\partial_{\tau} \mu \nabla u\right|_{2}^{2}+\right.\right. \\
& \left|\sqrt{\rho} \partial_{\tau} u\right|_{2}^{2}+|\nabla u|_{3}^{2}+(\mu-1)^{2}\left(\left|\nabla \partial_{\tau} u\right|_{2}^{2}+|\nabla u|_{2}^{2}\right)+k\left(|u|_{2}^{2}+\left|\partial_{t} u\right|_{2}^{2}\right)+ \\
& \left.\left.\left|\partial_{\tau} \mathcal{T}\right|_{2}^{2}+|f|_{2}^{2}+\left|\partial_{\tau} \rho\right|_{2}^{2}|f|_{3}^{2}+\left|\partial_{\tau} f\right|_{2}\right) d \tau\right]+\delta \int_{0}^{t}\left(\left|\nabla \partial_{\tau} v\right|_{2}^{2}+|v|_{2}^{2}\right) d \tau
\end{aligned}
$$

The estimate (4.15) implies

$$
\begin{aligned}
& \sqrt{\rho} \partial_{t} v \in L^{\infty}\left(0, T ; L^{2}(\Omega)\right), \quad \partial_{t} v \in L^{2}\left(0, T ; \tilde{H}^{1}(\Omega)\right), \\
& v \in L^{\infty}\left(0, T ; \tilde{H}^{1}(\Omega)\right) .
\end{aligned}
$$

Now we consider

$$
\begin{aligned}
& P \Delta v-k v=P\left(\rho \partial_{t} v+\rho u \cdot \nabla u-\nabla \cdot \mathcal{T}-\right. \\
& \nabla \cdot((\mu-1) \nabla u)-k u-\rho f) \equiv \mathcal{Z} .
\end{aligned}
$$

This is a Stokes system. Thanks to (4.2)-(4.8) and (4.16), we have

$$
\mathcal{Z} \in L^{2}\left(0, T ; \tilde{H}^{1}(\Omega)\right) \cap L^{\infty}\left(0, T ; L^{2}(\Omega)\right) .
$$

Then

$$
v \in L^{2}\left(0, T ; \tilde{H}^{3}(\Omega)\right) \cap L^{\infty}\left(0, T ; \tilde{H}^{2}(\Omega)\right) .
$$

Now the existence and uniqueness of the solution to the system (4.10) enables us to define the map $v=G u$ given by the composition of $g: u \rightarrow(\rho, \mu, F)$ and $h:(u, \rho, \mu, F) \rightarrow v$. The fixed point of $G$ is the solution of the system (2.6).

Let us consider the set

$$
\begin{gathered}
B=\left\{\phi \mid \sup \left(\|\phi\|_{L^{2}\left(0, T ; \tilde{H}^{3}(\Omega)\right)} ;\|\phi\|_{L^{\infty}\left(0, T ; \tilde{H}^{2}(\Omega)\right)}\right.\right. \\
\left.\left.\left\|\partial_{t} \phi\right\|_{L^{\infty}\left(0, T ; L^{2}(\Omega)\right)} ;\left\|\partial_{t} \phi\right\|_{L^{2}\left(0, T ; \tilde{H}^{1}(\Omega)\right)}\right) \leq r\right\}
\end{gathered}
$$

with

$$
r^{2}=\tilde{c} e^{\left\|v_{0}\right\|_{1}}\left(\left|\Delta v_{0}\right|_{2}^{2}+\left|\nabla \mu_{0} \nabla v_{0}\right|_{2}^{2}+\left|v_{0} \cdot \nabla v_{0}\right|_{2}^{2}+|\nabla \cdot \mathcal{T}(0)|_{2}^{2}+|f(0)|_{2}^{2}\right) / \alpha,
$$

where $\tilde{c} \geq 1$ is a suitable constant.

It is clear that $B$ is a compact set in $L_{l o c}^{2}\left(Q_{T}\right)$. As we are going to use a fixed point theorem, we have to show that $G B \subseteq B$ and $G$ is continuous in $B$ with respect to the norm in $L_{l o c}^{2}\left(Q_{T}\right)$.

Next we prove $G B \subseteq B$ for suitable $\bar{T}$. In fact, assuming $u \in B$, from (4.2)-(4.5), we have

$$
\begin{aligned}
& \alpha \leq \rho \leq 1 \\
& |\nabla \rho|_{q} \leq\left|\nabla \rho_{0}\right|_{q} e^{c r \sqrt{t}}, \quad(2 \leq q \leq 6) \\
& \|\rho\|_{2} \leq c e^{c r \sqrt{t}+t}\left\|\rho_{0}\right\|_{2}\left(1+c r \sqrt{t} e^{c r \sqrt{t}}\right) .
\end{aligned}
$$


The same estimates hold for $\mu$. Further

$$
\begin{aligned}
& |F| \leq\left|F_{0}\right| e^{r \sqrt{t}}, \\
& |\nabla F|_{p} \leq e^{c r \sqrt{t}}\left(\left|\nabla F_{0}\right|_{p}+\left|F_{0}\right|_{\infty} r \sqrt{t} e^{r \sqrt{t}}\right) \quad(2 \leq p \leq 6), \\
& \|F\|_{2} \leq c e^{c r \sqrt{t}}\left(\left\|F_{0}\right\|_{2}+r \sqrt{t} e^{c r \sqrt{t}}\left(\left|\nabla F_{0}\right|_{3}+\left|F_{0}\right|_{\infty}+\left|F_{0}\right|_{\infty} r \sqrt{t} e^{c r \sqrt{t}}\right)\right) .
\end{aligned}
$$

Therefore from $(4.14),(4.15),(4.19)$ and (4.20) we have

$$
\begin{aligned}
& \frac{1}{2}\left|\sqrt{\rho}(t) \partial_{t} v(t)\right|_{2}^{2}+\frac{1}{2} \int_{0}^{t}\left(\left|\nabla \partial_{\tau} v\right|_{2}^{2}+\frac{k}{2}\left|\partial_{\tau} v\right|_{2}^{2}\right) d \tau \leq \frac{1}{2}\left|\sqrt{\rho}(0) \partial_{t} v(0)\right|_{2}^{2}+ \\
& c_{\delta}\left[t r^{2} \sup _{0 \leq \tau \leq t}\left|\sqrt{\rho} \partial_{\tau} u\right|_{2}^{2}+t\left(r^{6}+c r^{4} e^{c r \sqrt{t}}\right)+t \frac{|1-\beta|^{2}}{2} r^{2}+\right. \\
& \left.k^{2} t r^{2}+\int_{0}^{t}\left|\partial_{\tau} f\right|_{2}^{2} d \tau+\sup _{0 \leq \tau \leq t}\left|\partial_{\tau} \rho\right|_{2}^{2} \int_{0}^{t}|f|_{3}^{2} d \tau\right]+ \\
& \delta t \sup _{0 \leq \tau \leq t}\left|\sqrt{\rho} \partial_{\tau} u\right|_{2}^{2}+\delta \int_{0}^{t}\left(\left|\nabla \partial_{t} v\right|_{2}^{2}+|v|_{2}^{2}\right) d \tau \leq r^{2}
\end{aligned}
$$

with suitable $\delta$ and $\bar{T}$. Now from (4.17) and (2.1) we find (to avoid tedious control of constants we assume for simplicity that $\left.\|v\|_{3} \leq\|(A+k) v\|_{1}\right)$

$$
\begin{aligned}
& \int_{0}^{\bar{T}}\|v\|_{3}^{2} d t \leq \int_{0}^{\bar{T}}\left(\|(A+k) v\|_{1}^{2} d t \leq\right. \\
& c \sup _{0 \leq t \leq \bar{T}}\left(|\nabla \rho|_{6}^{2}\left|\partial_{t} u\right|_{2}^{2}\right)\left(\int_{0}^{\bar{T}}\left\|\partial_{t} v\right\|_{1}^{2} d t\right)^{1 / 2}(\bar{T})^{1 / 2}+ \\
& c \int_{0}^{\bar{T}}\left(\left|\nabla \partial_{t} v\right|_{2}^{2}+|(\nabla \mu) \Delta u|_{2}^{2}+|\nabla(\nabla \mu) \nabla u|_{2}^{2}+|| \mathcal{T}||_{2}^{2}+\right. \\
& \left.|\nabla \rho|_{6}^{2}|u|_{\infty}^{2}|\nabla u|_{3}^{2}+|u|_{\infty}^{2}|\nabla(\nabla u)|_{2}^{2}+k\|u\|_{1}^{2}+|\nabla f|_{2}^{2}+|\nabla \rho|_{4}^{2}|f|_{4}^{2}\right) d t+ \\
& \int_{0}^{\bar{T}}|1-\beta|^{2}|\nabla \Delta u|_{2}^{2} d t \leq r^{2},
\end{aligned}
$$

for suitable $\bar{T}$ and $\beta$ (we denote with the same letter possibly different suitable $\bar{T}$ ). Whence (4.21), (4.22) imply

$$
G B \subseteq B .
$$

Now we prove the continuity of $G$ in $L_{l o c}^{2}\left(Q_{T}\right)$. First, we introduce for any fixed compact $A \subset \Omega$, such that $\sup _{x \in A}|x| \leq R\left(R>0\right.$ large enough), a cut-off function $\phi \in C^{1}$ such that $\phi \equiv 1$ on $A, \phi \geq 0$ on $\Omega$ and $|\nabla \phi| \leq \frac{1}{R}$ for $|x| \geq 2 R$. First we observe that if $\left\{u^{n}\right\} \subseteq B$, then there exists a subsequence (denoted again by $\left\{u^{n}\right\}$ ) such that as $n \rightarrow \infty$, $u^{n} \rightarrow u$ strongly in $L^{2}\left(0, \bar{T} ; \tilde{H}_{l o c}^{2}(\Omega)\right)$, weak ${ }^{*}$ in $L^{\infty}\left(0, \bar{T} ; \tilde{H}^{2}(\Omega)\right)$, and $\partial_{t} u^{n} \rightarrow \partial_{t} u$ weakly in $L^{2}\left(0, \bar{T} ; \tilde{H}^{1}(\Omega)\right)$. Let $\rho^{n}$ and $\rho$ be the solutions of

$$
\begin{gathered}
\partial_{t} \rho^{n}+\nabla \cdot\left(\rho^{n} u^{n}\right)=0 \quad \text { with } \quad \rho^{n}(0)=\rho_{0}, \\
\partial_{t} \rho+\nabla \cdot(\rho u)=0 \quad \text { with } \quad \rho(0)=\rho_{0},
\end{gathered}
$$

respectively. Analogously for $\left(\mu^{n}, \mu\right)$ and $\left(F^{n}, F\right)$. 
Now $\tau^{n}=\rho^{n}-\rho$ satisfies

$$
\partial_{t} \tau^{n}+u^{n} \cdot \nabla \tau^{n}+\left(u^{n}-u\right) \cdot \nabla \rho=0
$$

with $\tau^{n}(0)=0$. Multiplying (4.23) by $\tau^{n} \phi^{2}$ integrating over $Q_{T}$, and applying Gronwall lemma, we have

$$
\left|\tau^{n} \phi\right|_{2}^{2} \leq e^{c \bar{T}} \int_{0}^{\bar{T}}\left(\left|\phi\left(u^{n}-u\right) \cdot \nabla \rho\right|_{2}^{2}+\left|u^{n} \tau^{n} \nabla \phi\right|_{2}^{2}\right) d t .
$$

Bearing in mind (2.1), this implies that $\rho^{n} \rightarrow \rho$ strongly in $L^{\infty}\left(0, \bar{T} ; L^{2}(\tilde{\Omega})\right)$ for arbitrary $\tilde{\Omega} \subset A$, first passing to the limit $n \rightarrow \infty$ and then to the limit $R \rightarrow \infty$. The same convergence holds for $\left\{\mu^{n}\right\}$.

Concerning $F^{n}$ we have that $\mathcal{F}^{n}=F^{n}-F$ satisfies

$$
\partial_{t} \mathcal{F}^{n}+u^{n} \cdot \nabla \mathcal{F}^{n}+\left(u^{n}-u\right) \cdot \nabla F=\mathcal{F}^{n} \nabla u^{n}+F \nabla\left(u^{n}-u\right) .
$$

As for $\tau^{n}$, this implies that $F^{n} \rightarrow F$ strongly in $L^{\infty}\left(0, \bar{T} ; L_{l o c}^{2}(\Omega)\right)$.

Now let $v^{n}$ and $v$ be solutions of

$$
\begin{aligned}
& \rho^{n} \partial_{t} v^{n}+\rho^{n} u^{n} \cdot \nabla u^{n}-\Delta v^{n}+k\left(v^{n}-u^{n}\right)-\nabla \cdot \mathcal{T}^{n}- \\
& \rho^{n} f-\nabla \cdot\left(\left(\mu^{n}-1\right) \nabla u^{n}\right)+\nabla p^{n}=0,
\end{aligned}
$$

with $v^{n}(0)=v_{0}$, and

$$
\begin{aligned}
& \rho \partial_{t} v+\rho u \cdot \nabla u-\nabla \cdot \mathcal{T}-\Delta v+k(v-u)- \\
& -\rho f-\nabla \cdot((\mu-1) \nabla u)+\nabla p=0,
\end{aligned}
$$

with $v(0)=v_{0}$ and $p^{n}, p$ are the pressures obtained in the standard manner.

Subtracting (4.25) and (4.26), denoting $V^{n}=v^{n}-v, U^{n}=u^{n}-u, P^{n}=p^{n}-p$ we obtain

$$
\begin{aligned}
& \left(\rho^{n} \partial_{t} V^{n}+\tau^{n} \partial_{t} v+\rho^{n} U^{n} \cdot \nabla u^{n}+\tau^{n} u \cdot \nabla u^{n}+\rho u \cdot \nabla U^{n}-\right. \\
& \left.\tau^{n} f-\nabla \cdot \mathcal{T}^{n}+\nabla \cdot \mathcal{T}-\nabla \cdot\left(\left(\mu^{n}\right) \nabla U^{n}\right)-\nabla \cdot\left(\left(\mu^{n}-\mu\right) \nabla u\right)\right)- \\
& \Delta V^{n}+k V^{n}+\Delta U^{n}-k U^{n}+\nabla P^{n}=0 .
\end{aligned}
$$

Multiplying (4.27) by $\partial_{t} V^{n} \phi^{2}$ and integrating over $Q_{T}$ we find

$$
\begin{aligned}
& \int_{0}^{T}\left|\sqrt{\rho^{n}} \partial_{t} V^{n} \phi\right|_{2}^{2} d \tau+\frac{1}{2}\left|\nabla V^{n}(t) \phi\right|_{2}^{2} \leq \\
& \int_{0}^{\bar{T}}\left(-\tau^{n} \partial_{t} v-\rho^{n} U^{n} \cdot \nabla u^{n}-\tau^{n} u \cdot \nabla u^{n}-\rho u \cdot \nabla U^{n}+\tau^{n} f+\right. \\
& \left.\nabla \cdot\left(\mathcal{T}^{n}-\mathcal{T}\right)+\nabla \cdot\left(\mu^{n} \nabla U^{n}\right)+\nabla \cdot\left(\left(\mu^{n}-\mu\right) \nabla u\right)-(\Delta-k) U^{n}, \phi^{2} \partial_{t} V^{n}\right) d t+ \\
& \int_{0}^{t}\left(P^{n} \partial_{t} V^{n}, \phi \nabla \phi\right) d t+2 \sup _{t}\left|\nabla V^{n} V^{n} \phi \nabla \phi\right|_{2} .
\end{aligned}
$$

Thanks to

$$
\begin{gathered}
\left(\tau^{n}, \mu^{n}-\mu, \mathcal{F}^{n}\right) \rightarrow 0 \quad \text { strongly in } L^{\infty}\left(0, \bar{T} ; L_{l o c}^{2}(\Omega)\right), \\
U^{n} \rightarrow 0 \quad \text { strongly in } L^{2}\left(0, \bar{T} ; \tilde{H}_{l o c}^{1}(\Omega)\right)
\end{gathered}
$$

it is a routine matter to prove that $V^{n} \rightarrow 0$ strongly in $L_{l o c}^{2}\left(Q_{T}\right)$. Consequently, the map $G$ is continuous in $L_{l o c}^{2}\left(Q_{T}\right)$, and the existence of a local solution is completely proved. 
The proof of the uniqueness of the solution is obtained by the same procedure as that used for the continuity of $G$, considering $\left(\rho^{n}, \mu^{n}, F^{n}, v^{n}\right) \equiv\left(\rho^{1}, \mu^{1}, F^{1}, v^{1}\right)$ and $(\rho, \mu, F, v)$ as two solutions of (2.8) with the same data and without localization.

Theorem 3.6 is completely proved.

4.2. Proof of Theorem 3.7. Under the assumptions of Theorem 3.7, (4.16), the first two relations in (4.19) and (4.20) continue to hold. Bearing in mind that (4.17) is the Stokes system, we have, with $r$ as in Theorem 3.6,

$$
\begin{aligned}
& \int_{0}^{T}\|v\|_{2, q}^{2} d t \leq \int_{0}^{T}\left(\left|\partial_{t} v\right|_{q}^{2}+|u|_{\infty}^{2}|\nabla u|_{q}^{2}+k|u|_{q}^{2}+|f|_{q}^{2}+\right. \\
& \left.|\nabla \mathcal{T}|_{q}^{2}+|\nabla \mu \nabla u|_{q}^{2}+|\beta-1|^{2}|\Delta u|_{q}^{2}\right) d t \leq c \int_{0}^{T}|f|_{q}^{2} d t+c T\left(e^{c r \sqrt{T}}\left|\nabla F_{0}\right|_{2}^{2}+\right. \\
& \left.e^{c r \sqrt{T}} r^{2}+r^{6}\right)+c e^{-\left\|v_{0}\right\|_{1}} r^{2}+|\beta-1|^{2} r^{2} \leq r^{2}
\end{aligned}
$$

for suitable $T$ and $\beta$. We deduce immediately that $G B \subseteq B$. The continuity of $G$ is proved as in Theorem 3.6. Moreover, a uniqueness theorem holds. Theorem 3.7 is proved.

4.3. Proof of Theorem 3.8. First, to prove the existence of a solution of (4.1) we consider an approximation $\rho_{0}^{\epsilon}$ of $\rho_{0}$ such that

$$
\nabla \rho_{0}^{\epsilon} \in \tilde{H}^{1}(\Omega), \quad 0<\epsilon \leq \rho_{0}^{\epsilon} \leq 1, \quad \nabla \rho_{0}^{\epsilon} \rightarrow \nabla \rho_{0} \quad \text { as } \quad \epsilon \rightarrow 0
$$

strongly in $\tilde{H}^{1}(\Omega)$, and $\rho_{0} \leq \rho_{0}^{\epsilon}$. Then there exists a solution of (4.1) for the initial density $\rho_{0}^{\epsilon}$.

In what follows, we denote simply by $(v, \rho, \mu, F)$ the solution of (4.1) with the data $\rho_{0}^{\epsilon}$.

Bearing in mind that

$$
\begin{array}{r}
\frac{1}{2}\left|\sqrt{\rho}(0) \partial_{t} v(0)\right|_{2}^{2} \leq c\left(\left|\sqrt{\rho_{0}} v_{0} \cdot \nabla v_{0}\right|_{2}^{2}+\right. \\
\left.\left|\frac{\nabla \cdot\left(\mu_{0} \nabla v_{0}\right)+\nabla \cdot \mathcal{T}_{0}}{\sqrt{\rho}_{0}}\right|_{2}^{2}+|f(0)|_{2}^{2}\right)
\end{array}
$$

(4.14) gives

$$
\sqrt{\rho} \partial_{t} v \in L^{2}\left(Q_{T}\right) \cap L^{\infty}\left(0, T ; L^{2}(\Omega)\right), \partial_{t} \nabla v \in L^{2}\left(Q_{T}\right) .
$$

Furthermore, from (4.17)

$$
v \in L^{2}\left(\left(0, T ; H^{3}(\Omega) \cap \tilde{V}\right) \cap L^{\infty}\left(0, T ; \tilde{H}^{2}(\Omega)\right) .\right.
$$

Now we prove $G B \subseteq B$, where $B$ is as in the proof of Theorem 3.6 with

$$
r^{2}=\tilde{c} e^{\left\|v_{0}\right\|_{1}}\left(\left|\sqrt{\rho}_{0} v_{0} \cdot \nabla v_{0}\right|_{2}^{2}+\left|\left(\nabla \cdot\left(\mu_{0} \nabla v_{0}\right)+\nabla \cdot \mathcal{T}_{0}\right) / \sqrt{\rho}_{0}\right|_{2}^{2}+|f(0)|_{2}^{2}\right.
$$

$\tilde{c}>1$ a suitable constant.

From (4.27), as in Theorem 3.6, for suitable $\bar{T}_{\epsilon} \equiv \bar{T}$ and $\delta$, we have

$$
\sup _{0 \leq t \leq \bar{T}}\left|\sqrt{\rho} \partial_{t} v(t)\right|_{2}^{2}+\int_{0}^{\bar{T}}\left|\nabla \partial_{t} v(t)\right|_{2}^{2} d t \leq r^{2} e^{-\left\|v_{0}\right\|_{1}} .
$$


Now thanks to (4.21), we have

$$
\nabla\left(\rho \partial_{t} v\right)=\nabla \rho \partial_{t} v+\rho \nabla \partial_{t} v \in L^{2}\left(Q_{\bar{T}}\right)
$$

Now repeating the calculations to obtain (4.23), for suitable $\bar{T}$, and $\beta$, we get

$$
G B \subseteq B .
$$

As in Theorem 3.6, we have the continuity of $G$ in $L^{2}\left(Q_{\bar{T}}\right)$. The existence of a solution of (4.1) is proved. Now, for the solution $v^{\epsilon}$ the estimates (4.28) and (4.22) for $\left\|A v^{\epsilon}\right\|_{1}$ hold for a $\bar{T}$ which depends only upon the data and is independent of $\epsilon$. Consequently $v^{\epsilon}$ belongs to

$$
\begin{gathered}
\tilde{B}=\left\{\phi \mid \sup \left(\|\phi\|_{L^{2}\left(0, T ; H^{3}(\Omega) \cap V\right)} ;\|\phi\|_{L^{\infty}\left(0, T ; H^{2}(\Omega) \cap V\right)} ;\right.\right. \\
\left.\sup _{t}\left|\sqrt{\rho}\left(\phi+\partial_{t} \phi\right)\right|_{2}^{2},\left\|\partial_{t} \phi\right\|_{L^{2}\left(0, T ; H^{1}(\Omega)\right)} \leq r\right\},
\end{gathered}
$$

uniformly with respect to $\epsilon$ and $\rho^{\epsilon}$ satisfies (4.3) with $\alpha=0$ uniformly with respect to $\epsilon$. Hence, passing to the limit $\epsilon \rightarrow 0$, it is routine matter to obtain a solution $(v, \rho, \mu, F)$ of (2.8).

The uniqueness of the solution is proved in just the same manner as in Theorem 3.6. Theorem 3.8 is completely proved.

5. Discontinuous model. In this section we prove the existence of the discontinuous model. The proofs remain on the results in [9], [11], [12] and [13].

5.1. Proof of Theorem 3.10. The presence of the surface tension in the system (2.6) and the evolution equation of $F$ give a technical difficulty which leads to the introduction of a regularization through the addition of a viscosity term $\eta\left(A^{s}+I\right)$ with $s>3$, where $A^{s}$ is the operator associated to the bilinear form $((u, v))_{s}$ on the space $V^{s}$. We assume $s=4$. Moreover, for simplicity, assume $\Omega^{2}(0)$ is a bounded domain.

We thus introduce the regularized system

$$
\begin{aligned}
& \rho \partial v_{t}+\rho v \cdot \nabla v-\nabla \cdot(\mu \nabla v)+\eta\left(A^{s}+I\right) v-\nabla \cdot \mathcal{T}+\nabla \chi^{2} \mathcal{H}+\nabla p=f, \\
& \nabla \cdot v=0, \\
& K v(x, t)=0, \text { on } \partial \Omega, v=0 \text { at } \infty, v(x, 0)=v_{0}, \text { in } \Omega, \\
& \partial_{t} \chi+v \cdot \nabla \chi=0, \\
& \partial_{t} F+v \cdot \nabla F=\nabla v F .
\end{aligned}
$$

Here $K$ is the boundary operator associated to the bilinear form $((\cdot, \cdot))_{s}$ and $\chi^{2}(t), \mathcal{H}$ are the characteristic function and the mean curvature of the transformed domain $\Omega^{2}(t)$ obtained from $\Omega^{2}(0)$ in terms of the vector field $v$, respectively. Further we notice that, bearing in mind the form of $\rho(>0)$ and $\mu(>0)$, we consider the continuity equation for $\chi$ only.

The proof of Theorem 3.10 is divided into three steps.

STEP 1. Auxiliary problem. We consider the following system:

$$
\begin{aligned}
& \rho \partial_{t} v+\rho u \cdot \nabla v-\nabla \cdot(\mu \nabla v)+\eta\left(A^{4}+I\right) v-\nabla \cdot \mathcal{T}+\nabla \chi^{2} \mathcal{H}+\nabla p=f \\
& \nabla \cdot v=0
\end{aligned}
$$




$$
\begin{aligned}
& K v(x, t)=0, \text { on } \partial \Omega, v=0 \text { at } \infty, v(x, 0)=v_{0} \text { in } \Omega, \\
& \partial_{t} \chi+u \cdot \nabla \chi=0, \chi(0)=\chi_{0}, \\
& \partial_{t} F+u \cdot \nabla F=\nabla u F, \quad F(0)=F_{0} .
\end{aligned}
$$

$u, \rho, \mu, v(0), F_{0}$ are smooth given functions. $\chi^{2}, \mathcal{H}$ are the characteristic function and the mean curvature of the transformed domain $\Omega^{2}(t)$ obtained in terms of a given vector field $u$ as the set of points $x=x(y, t)$ such that

$$
\partial_{t} x=u(x, t), \quad x(y, 0)=y \in \Omega^{2}(0) .
$$

Moreover, we assume that $\chi_{0}$ is regular (it will be defined in step 2).

The existence of a solution of $(5.2)_{4}$ and $(5.2)_{5}$ is obtained as in Theorem 3.6.

Now we prove the existence of a solution of $(5.2)_{1}$. First we consider the existence of a strong solution to the system

$$
\begin{aligned}
& \partial_{t} v+A^{4} v+v=P f \\
& K v=0 \text { on } \partial \Omega, v=0 \text { at } \infty, \quad v(0)=v_{0} .
\end{aligned}
$$

Let

$$
\mathcal{F}=\left\{\phi \mid \phi \in L^{2}\left(0, T ; H^{8}(\Omega) \cap \tilde{V}\right), K(\phi)=0\right\}
$$

with the natural norm, and

$$
\mathcal{G}=\left\{\phi \mid \phi \in L^{2}\left(0, T ; \tilde{H}_{0}^{8}(\Omega) \cap V\right), \phi_{t} \in C\left([0, T] ; \tilde{H}^{8}(\Omega)\right), \phi(T)=0\right\} .
$$

We consider on $\mathcal{G}$ the norm

$$
\|\phi\|_{\mathcal{G}}=\|\phi\|_{\mathcal{F}}+\|\| \phi(0) \|_{4}
$$

We let

$$
\begin{aligned}
& E(v, \phi)=\int_{0}^{T}\left(-\left(v, \partial_{t}\left(A^{4}+I\right) \phi\right)+\left(A^{4} v+v,\left(A^{4}+I\right) \phi\right)\right) d t \\
& L(\phi)=\int_{0}^{T}\left(f,\left(A^{4}+I\right) \phi\right) d t+\left(v_{0},\left(A^{4}+I\right) \phi(0)\right) .
\end{aligned}
$$

First, $L(\phi)$ is a linear continuous form on $\mathcal{G}$ with respect to the norm $\|\cdot\|_{\mathcal{G}}$. Moreover, bearing in mind $\left|\left(A^{4}+I\right) \phi\right|_{2} \geq c\left|\|\phi \mid\|_{8}\right.$, we have

$$
\begin{aligned}
& E(\phi, \phi)=\int_{0}^{T}\left(\left(\left(-\partial_{t} \phi, \phi\right)\right)_{4}-\left(\partial_{t} \phi, \phi\right)+2\left|A^{2} \phi\right|_{2}^{2}+\left|A^{4} \phi\right|_{2}^{2}+|\phi|_{2}^{2}\right) d t \geq \\
& c\left(\left.\||| \phi(0)\|\right|_{4} ^{2}+\int_{0}^{T}\left(\left|A^{4} \phi\right|_{2}^{2}+\left|A^{2} \phi\right|_{2}^{2}+|\phi|_{2}^{2}\right) d t\right) \geq c\|\phi\|_{\mathcal{G}} .
\end{aligned}
$$

Then, from the Lax-Milgram theorem, there exists a $v \in \mathcal{F}$ such that

$$
E(v, \phi)=L(\phi)
$$

is satisfied for every $\phi \in \mathcal{G}$. Of course

$$
\|v\|_{\mathcal{F}} \leq c .
$$


Now if $g(t) \in C_{0}^{\infty}(\Omega)$ and $g(0)=g(T)=0$ and $\phi$ solution of $\left(A^{4}+I\right) \phi=P g(t)$ one has

$$
\begin{aligned}
& \left|\int_{0}^{T}\left(v(t), \partial_{t} g(t)\right) d t\right| \leq\left|\int_{0}^{T}\left(\left(A^{4} v(t)+v, g(t)\right)+(f, P g(t))\right) d t\right| \\
& \leq c\left(\int_{0}^{T}|g(t)|^{2} d t\right)^{1 / 2} .
\end{aligned}
$$

Hence $\partial_{t} v \in L^{2}\left(Q_{T}\right)$. By standard arguments, $v$ satisfies (5.3) a.e. in $Q_{T}$.

Now let

$$
\tilde{F}=\left\{\phi \mid \phi \in L^{2}\left(0, T ; H^{8}(\Omega) \cap \tilde{V}\right), \partial_{t} \phi \in L^{2}\left(Q_{T}\right), K(\phi)=0\right\}
$$

with the natural norm; let $\tilde{G}$ be the closure of the space

$$
G=\left\{\phi \mid \phi \in L^{2}\left(0, T ; \tilde{H}_{0}^{8}(\Omega) \cap V\right), \partial_{t} \phi \in C_{0}\left([0, T] ; \tilde{H}^{8}(\Omega)\right)\right\}
$$

in the norm

$$
\|\phi\|_{G}=\|\phi\|_{\tilde{F}}+\|\phi(0)\|_{4} .
$$

Now we prove the existence of a solution of $(5.2)_{1}$. We note that in the spaces considered $\nabla \chi^{2}$ is a smooth trace operator. We let

$$
\begin{aligned}
& E(v, \phi)= \\
& \int_{0}^{T}\left(\rho\left(\partial_{t} v+u \cdot \nabla v\right)-\nabla \cdot(\mu \nabla v)+\eta\left(A^{4}+I\right) v, \partial_{t} \phi+\eta A^{4} \phi+k \phi\right) d t+ \\
& \quad k\left(\left(v(0), A^{4} \phi(0)\right)+(v(0), \phi(0))\right), \\
& L(\phi)=\int_{0}^{T}\left(f-\mathcal{H} \nabla \chi^{2}+\nabla \cdot \mathcal{T}, \partial_{t} \phi+\eta A^{4} \phi+k \phi\right) d t+k\left(\left(v_{0}, A^{4} \phi(0)\right)+\left(v_{0}, \phi(0)\right)\right) .
\end{aligned}
$$

Here $k$ is a positive suitable number.

We remark that if $\phi \in L^{2}(\Omega)$ and $\nabla \cdot \phi=0$ then

$$
\|\phi n\|_{H^{-1 / 2}(\partial \Omega)} \leq\|\phi\|_{L^{2}(\Omega)} .
$$

Then $L(\phi)$ is a linear continuous form on $\tilde{G}$ with respect to the norm $\|\cdot\|_{\tilde{G}}$.

Moreover, bearing in mind that $\left|\left(A^{4}+I\right) \phi\right|_{2} \geq c|||\phi|||_{8}$, and $\rho, \mu$ satisfy the transport equation, we have

$$
\begin{aligned}
& E(\phi, \phi) \geq \\
& \int_{0}^{T}\left(\frac{3}{4}\left|\sqrt{\rho} \partial_{t} \phi\right|_{2}^{2}+\eta^{2}\left|A^{4} \phi\right|_{2}^{2}+\eta\left(\rho \partial_{t} \phi, A^{4} \phi\right)-\frac{3}{4}|\nabla \cdot(\mu \nabla \phi)|_{2}^{2}+\right. \\
& \left.\left(\rho u \cdot \nabla \phi, \partial_{t} \phi+\eta A^{4} \phi\right)+\left(k \eta+\eta^{2}\right)\left|A^{2} \phi\right|_{2}^{2}+k \eta|\phi|_{2}^{2}+k|\sqrt{\mu} \nabla \phi|_{2}^{2}\right) d t+ \\
& \frac{1}{2} \int_{Q_{T}} \nabla \cdot(u \mu)|\nabla \phi|^{2} d x d t+\frac{1}{2}\left(|\sqrt{\mu(T)} \nabla \phi(T)|_{2}^{2}-|\sqrt{\mu(0)} \nabla \phi(0)|_{2}^{2}\right)+ \\
& \frac{k}{2}\left(|\sqrt{\rho}(T) \phi(T)|_{2}^{2}-|\sqrt{\rho}(0) \phi(0)|_{2}^{2}\right)+\frac{\eta}{2}\left(\left|A^{2} \phi(T)\right|_{2}^{2}+|\phi(T)|_{2}^{2}-\left|A^{2} \phi(0)\right|_{2}^{2}-|\phi(0)|_{2}^{2}\right)+ \\
& k\left(\left|A^{2} \phi(0)\right|_{2}^{2}+|\phi(0)|_{2}^{2}\right) \geq c\|\phi\|_{G}^{2},
\end{aligned}
$$

for suitable $k$. Then, thanks to the Lax-Milgram theorem, there exists a $v \in \tilde{F}$ such that 


$$
E(v, \phi)=L(\phi)
$$

is satisfied for every $\phi \in \tilde{G}$.

Now let $\bar{\phi}$ be the solution of the problem

$$
\begin{aligned}
& \partial_{t} \bar{\phi}+\eta A^{4} \bar{\phi}+k \bar{\phi}=0 \text { in } Q_{T}, \\
& \bar{\phi}(0)=h(x) \text { in } \Omega, K(\bar{\phi})=0 \text { on } \partial \Omega, \bar{\phi}=0 \text { at } \infty .
\end{aligned}
$$

with $h(t)$ smooth enough, and $\nabla \cdot h=0$. Replacing in (5.5) $\phi$ with $\bar{\phi}$ we have

$$
\left(v(0),\left(A^{4}+I\right) h\right)=\left(v_{0},\left(A^{4}+I\right) h\right),
$$

which implies that $v(0)=v_{0}$.

Now let $\bar{\phi}$ be the solution of the problem

$$
\begin{aligned}
& \partial_{t} \bar{\phi}+\eta A^{4} \bar{\phi}+k \bar{\phi}=g \quad \text { in } \quad Q_{T}, \\
& \bar{\phi}(0)=0 \text { in } \Omega, K(\bar{\phi})=0 \text { on } \partial \Omega, \bar{\phi}=0 \text { at } \infty .
\end{aligned}
$$

with $g$ smooth enough and $\nabla \cdot g=0$. Replacing in (5.5) $\phi$ with $\bar{\phi}$ we have

$$
\int_{0}^{T}\left(\rho\left(\partial_{t} v+u \cdot \nabla v\right)-\nabla \cdot(\mu \nabla v)+\eta\left(A^{4}+I\right) v-\nabla \cdot \mathcal{T}-f+\mathcal{H} \nabla \chi^{2}, g\right) d t=0 .
$$

This implies that $v$ satisfies $(5.2)_{1}$ a.e. in $Q_{T}$.

SteP 2. Approximate problem. We consider the following system:

$$
\begin{aligned}
& \rho \partial_{t} v+\rho v_{\epsilon} \cdot \nabla v-\nabla \cdot(\mu \nabla v)+\eta\left(A^{4}+I\right) v-\nabla \cdot \mathcal{T}_{\epsilon}+\nabla p=f-\mathcal{H} \nabla \chi^{2}, \\
& \nabla \cdot v=0, \\
& K v(x, t)=0, \text { on } \partial \Omega, v=0 \text { at } \infty, \\
& \partial_{t} \chi+v_{\epsilon} \cdot \nabla \chi=0, \\
& \partial_{t} F+\bar{v}_{\epsilon} \cdot \nabla F=\nabla v_{\epsilon} F,
\end{aligned}
$$

with the initial conditions

$$
\chi_{\eta}(0)=\left(\chi_{0}\right)_{\eta} \equiv \chi_{0}^{\eta}, \quad v(0)=\left(v_{0}\right)_{\eta},
$$

where $v_{\epsilon}, \mathcal{T}_{\epsilon},\left(\chi_{0}\right)_{\eta},\left(v_{0}\right)_{\eta}$, are regularizations of $v, \mathcal{T}, \chi_{0}, v_{0}$, respectively. $v_{\epsilon}, \mathcal{T}_{\epsilon}$ are obtained by convolution. Further $\left(\chi_{0}\right)_{\eta}=\chi(0)$ on $\Omega^{1}(0)\left(\operatorname{supp}\left(\chi_{0}\right)_{\eta} \equiv \Omega_{\eta}^{1}(0) \subset \Omega^{1}(0)\right.$ is bounded, measure $\left(\Omega_{\eta}^{1}(0) \cap \Omega^{2}(0) \leq c \eta\right.$ and $\Omega_{\eta}^{1}(0) \rightarrow \Omega^{1}(0)$ as $\left.\eta \rightarrow 0\right)$, and $\mathcal{H}_{\epsilon} \equiv \mathcal{H}, \chi_{\epsilon}^{2} \equiv$ $\chi^{2}$ are the mean curvature and the characteristic function of the transformed domain $\Omega_{\epsilon}^{2}(t)$ obtained from $\Omega^{2}(0)$ in terms of the vector field $v_{\epsilon}$, respectively. Moreover, $\bar{v}_{\epsilon}$ is obtained by truncation of $v$ in $\Omega_{\epsilon}=\{x \in \Omega$, dist $(x, \partial \Omega)>2 \epsilon\}$ and by convolution with parameter $\epsilon$ (see [5] appendix A) so that the homogeneous Dirichlet boundary condition is satisfied. We recall that $\rho$ and $\mu$ are regular function of $\chi$.

Of course, we have

$$
\begin{aligned}
& 0 \leq\left(\chi_{0}\right)_{\eta}, \\
& \left(\chi_{0}\right)_{\eta} \rightarrow \chi_{0} \quad \text { in } L_{l o c}^{p}(\Omega) \quad(1 \leq p<\infty), \\
& \left(v_{0}\right)_{\eta} \rightarrow v_{0} \quad \text { in } L^{2}(\Omega) .
\end{aligned}
$$


We prove the existence of a solution of (5.6) by a fixed point argument. Let $B$ a convex set in $L^{2}(0, T ; \tilde{V})$ defined by

$$
B=\left\{u \mid u \in L^{2}(0, T ; \tilde{V}),\|u\|_{L^{2}(0, T ; \tilde{V})} \leq C\right\},
$$

with suitable $C$. We follow the procedure of Theorem 3.6. First we consider the solution, for fixed $\eta,\left(v^{\epsilon}, \chi^{\epsilon}, F^{\epsilon}\right) \equiv(v, \chi, F)$ of the system

$$
\begin{aligned}
& \rho \partial_{t} v+\rho u_{\epsilon} \cdot \nabla v-\nabla \cdot(\mu \nabla v)+\eta\left(A^{4}+I\right) v-\nabla \cdot \mathcal{T}_{\epsilon}+\nabla p=f-\mathcal{H} \nabla \chi^{2}, \\
& \nabla \cdot v=0, \\
& K v(x, t)=0, \text { on } \partial \Omega, v=0 \text { at } \infty, v(x, 0)=v_{0}, \\
& \partial_{t} \chi+u_{\epsilon} \cdot \nabla \chi=0, \chi(0)=\chi_{0}^{\eta}, \\
& \partial_{t} F+\bar{u}_{\epsilon} \cdot \nabla F=\nabla u_{\epsilon} F, F(0)=F_{0},
\end{aligned}
$$

with $u \in B$ and $\chi^{2}, \mathcal{H}$ are obtained using the vector field $u_{\epsilon}$.

First the existence of a unique solution $\chi$ of the transport equation follows from the method of characteristics as in Theorem 3.6. The explicit formula for $\chi$ is

$$
\chi(x, t)=\chi_{0}^{\eta}(y(0, x, t)) .
$$

Obviously $0 \leq \chi \leq c$ and $\chi \in L^{\infty}\left(0, T ; \tilde{H}^{2}(\Omega)\right)$ and $\partial_{t} \chi \in L^{2}\left(Q_{T}\right)$. Furthermore

$$
\|\chi(t)\|_{L^{2}(\Omega)}=\left\|\chi_{0}^{\eta}\right\|_{L^{2}(\Omega)} .
$$

Analogously we obtain the existence of $F$ solution of $(5.7)_{4}$. Then a solution $v$ of $(5.7)_{1}$ can be obtained as in step 1 .

Now we can define the map $G: u \rightarrow v$ given by the composition of $g: u \rightarrow(\chi, F)$ and $h:(F, \chi, u) \rightarrow(v)$.

The fixed point of $G$ is a solution of (5.7). For this we need a priori estimates. We multiply $(5.7)_{1}$ by $v$, integrate over $\Omega$, and obtain

$$
\begin{aligned}
& \int_{\Omega} \frac{1}{2}\left(\rho \partial_{t}|v|^{2}+\eta\left(v A^{4} v+v^{2}\right) d x+\int_{\Omega}\left(\frac{1}{2} \rho u_{\epsilon} \cdot \nabla|v|^{2}+|\sqrt{\mu} \nabla v|^{2}\right) d x=\right. \\
& \int_{\Omega}\left(f v-\nabla \chi^{2} \mathcal{H} v-\mathcal{T}_{\epsilon} \nabla v\right) d x .
\end{aligned}
$$

Bearing in mind the continuity equation and integrating over $(0, t)$, we have

$$
\begin{aligned}
& |\sqrt{\rho} v(t)|_{2}^{2}+\int_{0}^{t}\left(|\sqrt{\mu} \nabla v|_{2}^{2}+\eta\left|A^{2} v\right|_{4}^{2}+|v|_{2}^{2}\right) d t \leq \\
& c\left(\int_{0}^{t}|f|_{2}^{2} d t+T \int_{0}^{T}\|\mathcal{H}\|_{H^{1 / 2}(\Gamma(t))}^{2} d t+T\left|\mathcal{T}_{\epsilon}\right|_{\infty}^{2}\right)+|\sqrt{\rho(0)} v(0)|_{2}^{2}
\end{aligned}
$$

hence

$$
\sup _{0 \leq t \leq T}|v(t)|_{2}^{2}+\|v\|_{L^{2}\left(0, T ; \tilde{V} \cap H^{4}\right)}^{2} \leq c+T C c_{\epsilon, \eta},
$$

where $c$ is independent of $(C, \epsilon, \eta)$.

Now we choose $C>c$ and $T=\bar{T}$ sufficiently small so that $G B \subseteq B$. We multiply $(5.7)_{1}$ by $\partial_{t} v$, integrate over $\Omega$ and obtain

$$
\left|\sqrt{\rho} \partial_{t} v\right|_{2}^{2}+\frac{1}{2} \frac{d}{d t}\left(|\sqrt{\mu} \nabla v|_{2}^{2}+\eta\left(\left|A^{2} v\right|_{2}^{2}+|v|_{2}^{2}\right)\right) \leq
$$




$$
\begin{aligned}
& c\left(|f|_{2}^{2}+\|\mathcal{H}\|_{H^{1 / 2}(\Gamma(t))}^{2}+\left\|u_{\epsilon}\right\|_{L^{\infty}(\Omega)}^{2}|\nabla v|_{2}^{2}+\right. \\
& \left.\left|\nabla \cdot \mathcal{T}_{\epsilon}\right|_{2}^{2}+\left\|\partial_{t} \mu\right\|_{L^{\infty}(\Omega)}|\nabla v|_{2}^{2}\right) .
\end{aligned}
$$

We deduce from Gronwall's lemma that

$$
\int_{0}^{t}\left|\partial_{t} v\right|_{2}^{2} d t+|\nabla v(t)|_{2}^{2}+\eta\left(|v(t)|_{2}^{2}+\left|A^{2} v(t)\right|_{2}^{2}\right) \leq c_{\epsilon, \eta}
$$

Consequently the map $G$ is compact in $L^{2}\left(0, T ; L_{l o c}^{2}(\Omega)\right)$.

The fixed point of $G$ is the solution of $(5.7)$ in $(0, \bar{T})$. Thanks to $(5.7)_{5}$ we get

$$
\int_{\Omega} \nabla \cdot \mathcal{T}_{\epsilon} v d x=\int_{\Omega} \mathcal{T} \nabla v_{\epsilon} d x=d_{t} \int_{\Omega} \mathcal{W} d x
$$

holds.

Consequently we have the energy estimate

$$
\begin{aligned}
& \sup _{t}\left(|\sqrt{\rho} v(t)|_{2}^{2}+\int_{\Omega} \mathcal{W} d x\right)+\int_{0}^{t}\left(|\sqrt{\mu} \nabla v|_{2}^{2}+\eta\left(\left|A^{2} v\right|_{2}^{2}+|v|_{2}^{2}\right) d \tau \leq\right. \\
& c\left(|\sqrt{\rho}(0) v(0)|_{2}^{2}+\int_{\Omega} \mathcal{W}_{0} d x+\int_{0}^{t}|f|_{2}^{2} d \tau\right) .
\end{aligned}
$$

It is a routine matter to prove that the solution $(\chi, F, v)$ exists in $(0, T)$, and satisfies (5.7) a.e. in $\Omega$.

SteP 3. A priori estimates and limit $(\epsilon, \eta) \rightarrow 0$. From the results of step 2 we have the estimate

$$
\sup _{0 \leq t \leq T}\left(|v(t)|_{2}^{2}+\int_{\Omega} \mathcal{W} d x\right)+\eta\|v\|_{L^{2}\left(0, T ; \tilde{V} \cap H^{8}\right)}^{2}+\left\|\partial_{t} v\right\|_{L_{Q_{T}}} \leq c
$$

where $c$ depends on $\eta$ only. Consequently we can pass to the limit $\epsilon \rightarrow 0$ in (5.7) and obtain a solution $\left(v_{\eta}, \chi_{\eta}, F_{\eta}\right) \equiv(v, \chi, F)$ of $(5.1)$.

Now we prove the existence of a solution of (2.6).

From the transport equation and multiplying $(5.1)_{1}$ by $v$, integrating over $\Omega_{T}$ and bearing in mind (2.3), we obtain the estimates

$$
\begin{aligned}
& |\sqrt{\rho} v(t)|^{2}+\int_{\Omega} \mathcal{W}(t) d x+\int_{0}^{t}\left(|\sqrt{\mu} \nabla v|^{2}+\eta\left(\left|A^{2} v\right|_{2}^{2}+|v|_{2}^{2}\right) d t+\right. \\
& \operatorname{meas}(\Gamma(t)) \leq c \\
& 0 \leq \chi_{\eta} \leq c,\left\|\chi_{\eta}(t)\right\|_{L^{2}(\Omega)}=\left\|\chi_{\eta}(0)\right\|_{L^{2}(\Omega)} .
\end{aligned}
$$

The constant $c$ is independent of $\eta$. An immediate consequence of the above estimates is that the domains $\left(\Omega_{\eta}^{2}\right)_{T}=\cup\left(\Omega_{\eta}^{2}(t) \times t\right)$ have perimeters uniformly bounded with respect $\eta$. This implies that there exists a measurable set $\Omega_{T}^{2} \subset Q_{T}$ such that the characteristic functions $\chi_{\eta}^{2}$ of $\left\{\left(\Omega_{\eta}^{2}\right)_{T}\right\}$, up to a subsequence, converge to the characteristic function $\chi^{2}$ of $\Omega_{T}^{2}$, i.e.

$$
\chi_{\eta}^{2} \rightarrow \chi^{2} \text { in } L^{2}\left(Q_{T}\right)
$$

and

$$
P\left(\Omega_{T}^{2}\right) \leq \liminf P\left(\Omega_{\eta}^{2}\right)_{T}
$$


Thanks to

$$
\left|\int_{Q_{T} \cap\left(\Omega_{\eta}^{2}\right)_{T}} \chi_{\eta}^{1} d x\right| \leq c \eta
$$

we get

$$
\chi_{\eta}^{1} \rightarrow \chi^{1} \quad \text { strongly } \quad \text { in } \quad L_{l o c}^{2}\left(Q_{T}\right)
$$

Here $\chi^{1}=1-\chi^{2}$.

From (2.4)

$$
\left|\int_{0}^{T}\left(\nabla \chi_{\eta}^{2} \mathcal{H}_{\eta}, \phi\right) d t\right| \leq c \sup _{t}(\operatorname{meas}(\Gamma(t))) \int_{0}^{T} \sup _{x}|\nabla \phi| d t .
$$

for any $\phi \in L^{\infty}\left(0, T ; C_{0}^{\infty}(\Omega)\right)$. This implies $\left\{\nabla \chi_{\eta}^{2} \mathcal{H}_{\eta}\right\}$ is bounded in sense of distributions. Hence $\left\{\nabla \chi_{\eta}^{2} \mathcal{H}_{\eta}\right\}$ has a limit for $\eta \rightarrow 0$ that we will denote $\mathcal{H}$.

Now collecting the above arguments, we obtain as in [12], the estimate

$$
\left\|\partial_{t} P_{\Omega}\left(\rho_{\eta} v_{\eta}\right)\right\|_{L^{2}\left(0, T ; \tilde{H}^{-3}(\Omega)\right)} \leq c,
$$

where $c$ is independent of $\eta$ and $P_{\Omega}$ is the projection operator onto the solenoidal space $H(\Omega)$.

The above estimates permit us to prove that $\left\{v_{\eta}\right\}$ is a compact set in $L_{l o c}^{2}\left(Q_{T}\right)$. In fact, let $K \subset \Omega$ be an open bounded domain and $K^{\delta}$ the $\delta$-dilation of $K$. Let $\theta_{\delta} \equiv \theta \in C_{0}^{\infty}(\Omega)$, $\operatorname{Supp} \theta \subset K^{\delta}, \theta \equiv 1$ on $K$. Let $w_{\delta, \eta} \equiv w$ be the solution in $\tilde{K}=K^{\delta} \backslash K$ of

$$
\nabla \cdot w=\nabla \cdot\left(\theta\left(v_{\eta}-v\right)\right), w=0 \text { on } \partial \tilde{K} .
$$

Here $v$ is the weak limit of $\left\{v_{\eta}\right\}$ in $L^{2}(0, T ; \tilde{V})$.

We set $h_{\eta}=\theta\left(v_{\eta}-v\right)-w(w$ is extended by 0 in $K)$. We notice that $\nabla \cdot h_{\eta}=0$ and $h_{\eta} \in L^{2}\left(0, T ; H_{0}^{1}\left(K^{\delta}\right)\right)$.

Now we prove, for $\eta \rightarrow 0$,

$$
\int_{K_{T}}\left(\left(\rho v^{2}\right)_{\eta}-(\rho v)_{\eta} v\right) d x d t=\int_{K_{T}} h_{\eta}(\rho v)_{\eta} d x \rightarrow 0 .
$$

Here $K_{T}=K \times(0, T)\left(\right.$ and $\left.K_{T}^{\delta}=K^{\delta} \times(0, T)\right)$. Now

$$
\int_{K_{T}} h_{\eta}(\rho v)_{\eta} d x=\int_{K_{T}^{\delta}} h_{\eta}(\rho v)_{\eta} d x-\int_{\tilde{K}_{T}} h_{\eta}\left(\rho v_{\eta}\right) d x d t .
$$

The first integral on the right-hand side tends to zero as $\eta \rightarrow 0$ because $h_{\eta} \rightarrow$ 0 weakly in $L^{2}\left(0, T ; H_{0}^{1}\left(K^{\delta}\right)\right)$ and $P_{K^{\delta}}(\rho v)_{\eta} \rightarrow P_{K^{\delta}} \rho v$ strongly in $L^{2}\left(0, T ; H^{-1}\left(K^{\delta}\right)\right)$. Moreover, the second integral satisfies

$$
\left|\int_{\tilde{K}_{T}} h_{\eta}(\rho v)_{\eta} d x d t\right| \leq c \delta^{\alpha}
$$

with $c$ independent of $\eta$ and $\alpha$ a positive number. (5.15) is proved.

The estimates $(5.11),(5.14)$ and $(5.15)$ permit us to deduce that there exists a subsequence, still denoted by $\left\{\left(F_{\eta}, \chi_{\eta}, v_{\eta}\right)\right\}$, such that

$v_{\eta} \rightarrow v$ weakly in $L^{2}(0, T ; \tilde{V})$, strongly in $L_{l o c}^{2}\left(Q_{T}\right)$,

$\chi_{\eta} \rightarrow \chi$ weak $^{*}$ in $L^{\infty}\left(Q_{T}\right)$ and strongly in $L_{l o c}^{2}\left(Q_{T}\right)$,

$\rho\left(\chi_{\eta}\right) v_{\eta} \rightarrow \rho(\chi) v$ weak $^{*}$ in $L^{\infty}\left(0, T ; L^{2}(\Omega)\right)$, 


$$
\begin{aligned}
& \mu\left(\chi_{\eta}\right) \nabla v_{\eta} \rightarrow \mu(\chi) \nabla v \text { weakly in } L^{2}\left(Q_{T}\right), \\
& \rho\left(\chi_{\eta}\right)\left(v_{i}\right)_{\eta}\left(v_{j}\right)_{\eta} \rightarrow \rho(\chi) v_{i} v_{j} \text { weakly in } L^{p}\left(Q_{T}\right), p>1, \\
& F_{\eta} \rightarrow F \text { weakly in } L^{2}\left(Q_{T}\right), \\
& \chi_{\eta}^{2} F_{\eta} \rightarrow \chi^{2} F \text { weakly in } L^{2}\left(Q_{T}\right), \\
& \mathcal{T} \rightarrow \Lambda \text { in the sense of measure. }
\end{aligned}
$$

In subsection 4.4 we noticed that the localization of $F$ is obtained by localizing the initial data $F_{0}$. This procedure can produce some singular effect on the evolution of $\nabla \cdot F$, in general. For example $\nabla \cdot F$ can be a measure. But the assumption $n \cdot F_{0}=0$ on $\Gamma(0)$ circumvents this eventuality and implies that $\nabla \cdot\left(\chi^{2}(0) F_{0}\right)=\chi^{2}(0) \nabla \cdot F_{0}$. So we get $\left\{\nabla \cdot F_{\eta}^{T}\right\}$ is uniformly bounded in $L^{\infty}\left(Q_{T}\right)$. Thanks to $(5.16)$, we get

$$
\begin{gathered}
\int_{0}^{T} \int_{\Omega} \nabla v_{\eta} F_{\eta} \psi d x d t=-\int_{0}^{T}\left(v_{\eta} \nabla \cdot F_{\eta}^{T} \psi+v_{\eta} F_{\eta} \nabla \psi\right) d x d t \rightarrow \\
-\int_{0}^{T}\left(v \nabla \cdot F^{T} \psi+v F \nabla \psi\right) d x d t, \text { as } \eta \rightarrow 0,
\end{gathered}
$$

for any smooth tensor $\psi$ with compact supports. Now multiplying the equations in (5.2) by the respective test functions with compact support, integrating on $Q_{T}$ and passing to the limit $\eta \rightarrow 0$, we get that $(v, \chi, F, \Lambda)$ is a weak-measure solution of (2.6).

5.2. Proof of Theorem 3.11. The proof of Theorem 3.11 follows the procedure of Theorem 3.10. The main changes consist in

$$
d_{t}\left|F_{\eta}\right|^{2}=2\left(d_{t} F_{\eta}\right) \cdot F_{\eta}=2\left(W_{\eta}(v) F_{\eta}\right) \cdot F_{\eta}=0,
$$

and in the form of the energy estimate

(5.17) implies that

$$
\begin{aligned}
& \sup _{t}|\sqrt{\rho} v(t)|_{2}^{2}+\int_{0}^{t}\left(|\sqrt{\mu} \nabla v|_{2}^{2}+\eta\left(\left|A^{2} v\right|_{2}^{2}+|v|_{2}^{2}\right)\right) d \tau+\text { meas } \Gamma(t) \leq \\
& c\left(|\sqrt{\rho}(0) v(0)|_{2}^{2}+\operatorname{meas} \Gamma(0)+\sup _{t}|\mathcal{T}|_{2}^{2}+\int_{0}^{t}|f|_{2}^{2} d \tau\right) .
\end{aligned}
$$

$$
\|F(t)\|_{L^{2}(\Omega)}=\left\|F_{0}\right\|_{L^{2}(\Omega)} \forall t>0 ; F \in L^{\infty}\left(0, T ; L^{q}(\Omega)\right) \forall q>1 .
$$

Now $|F|^{2}$ satisfies the transport equation and thanks to the compactness result on transport equation in [5], $\left\{F_{\eta}\right\}$ is a compact set in $L_{l o c}^{q}\left(Q_{T}\right)$ for any $q \geq 1$. With the above results in hand, we get

$$
\int_{0}^{T} \int_{\Omega} W\left(v_{\eta}\right) F_{\eta} \psi d x d t \rightarrow \int_{0}^{T} \int_{\Omega} W(v) F \psi d x d t, \quad \text { as } \eta \rightarrow 0
$$

for any smooth tensor $\psi$ with compact support. Theorem 3.11 follows immediately from Theorem 3.10 .

5.3. Proof of Theorem 3.12. The core of the change of the proof of Theorem 3.12 with respect to Theorem 3.11 remains on the solution of

$$
\nabla \cdot F_{\eta}^{T}=g_{\eta} \quad \text { or } \quad \partial_{x_{i}}\left(F_{\eta}\right)_{i j}=\left(g_{\eta}\right)_{j},
$$

where $g_{\eta}$ is the solution of the transport equation $(2.7)_{4}$ in $L^{\infty}\left(Q_{T}\right)$. 
From Bogovskiı̌'s results (see [1]), there exists a solution $F_{\eta} \in W_{0}^{1, q}(\Omega)$ for any $q>1$ such that

$$
\left|\nabla F_{\eta}^{T}\right|_{q} \leq c\left|g_{\eta}\right|_{q}
$$

Moreover, we get

$$
\nabla \cdot \partial_{t} F_{\eta}^{T}=\partial_{t} g_{\eta} \in L^{\infty}\left(0, T ; H^{-1}(\Omega)\right)
$$

hence

$$
\left\|\partial_{t} F_{\eta}\right\|_{L_{l o c}^{2}\left(Q_{T}\right)} \leq c\left\|\partial_{t} g_{\eta}\right\|_{L^{2}\left(0, T ; H^{-1}(\Omega)\right)} .
$$

The above estimates imply that $\left\{F_{\eta}\right\}$ is a compact set in $L_{l o c}^{q}\left(Q_{T}\right)$. Now, the proof of Theorem 3.12 follows the arguments of Theorem 3.11 .

\section{References}

[1] M. E. Bogovski, Solution of some vector analysis problems connected with operators div and grad. Trudy Sem. Sobolev. 80 (1980), 5-40.

[2] L. Cattabriga, Su un problema al contorno relativo al sistema di equazioni di Stokes. Rend. Semin. Mat. Univ. Padova 31 (1961), 1-33.

[3] CJ. Y. Chemin and N. Masmoudi, About lifespan of regular solutions of equations related to viscoelastic fluids, SIAM J. Math. Anal. 33 (2001), 84-112.

[4] J.G. Heywood, The Navier-Stokes equations: On the existence, regularity and decay of solutions, Indiana Univ. Math. J. 40 (1990), 639-681.

[5] P. L. Lions, Mathematical Topics in Fluid Mechanics, Vol. I, Clarendon Press, Oxford, 1996.

[6] C. Liu and N. F. Walkington, An Eulerian description of fluids containing visco-elastic particles, Arch. Rational Mech. Anal. 159 (2001), 229-252.

[7] C. Guillopé and J. C. Saut, Existence results for the flow of viscoelastic fluids with a differential constitutive law, Nonlinear Analysis, 15 (1990), 849-869.

[8] C. Guillopé and J. C. Saut, Mathematical problems arising in differential models for viscoelastic fluids, in: J. F. Rodrigues and A. Sequeira (eds.), Mathematical Topics in Fluid Mechanics, Pitman Research Notes in Mathematics 274 (1992), 64-92.

[9] G. Nespoli and R. Salvi, On the existence of two-phase problem for incompressible flow, Quaderni di Matematica 4, P. Maremonti (ed.), 1999, 247-268.

[10] M. Renardy, Mathematical Analysis of Viscoelastic Flows, CBMS-NSF Regional Conf. Series in Applied Mathematics, 2000.

[11] R. Salvi, On the existence of weak solutions of boundary value problems in a diffusion model for an inhomogeneous liquid in regions with moving boundaries, Portugaliae Math. 43 (1985-86), 213-233.

[12] R. Salvi, On the existence of free surface problem for viscous incompressible flow, in: P. G. Galdi and R. Rannacher (eds.), Quaderni di Matematica 10 (2002), 249-275.

[13] R. Salvi, Existence and uniqueness results for non-Newtonian fluids of Oldroyd type, Int. Rev. Pure and Appl. Math. (IRPAM), (2005), 159-212.

[14] R. Salvi and I. Straskraba, Global existence for viscous compressible fluids and their behavior as $t \rightarrow \infty$, J. Fac. Sci. Univ. Tokyo Sect. IA Math. 40 (1993) 249-275.

[15] J. Sokolowski and J. P. Zolesio, Introduction to Shape Optimization, Springer Series in Computational Mathematics 16 (1992). 